\title{
The Metal Complexes of Peptides and Related Compounds
}

\author{
1. Copper(II) Complexes of O-Phosphorylserylglutamic Acid \\ in Aqueous Solution
}

\author{
RAG NAR ÖSTERBERG
}

Department of Medical Biochemistry, University of Göteborg, Göteborg, Sweden

\begin{abstract}
The complex formation between copper(II) and O-phosphorylserylglutamic acid has been studied potentiometrically in $0.15 \mathrm{M} \mathrm{K}\left(\mathrm{NO}_{3}\right)$ medium at $25^{\circ}$, using glass and copper amalgam electrodes. Species of type $\mathrm{MH}_{q}$ A with $q$ equal to $2,1,0,-1$ dominate in the concentration ranges investigated (Table 4). In addition, the existence of some $\mathrm{MH}_{q \mathrm{~A}_{2}},(1,2,2),(1,0,2)$, is indicated.

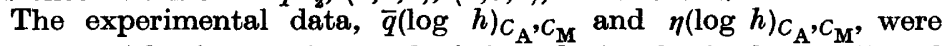
first treated by integration methods in order to obtain the free ligand concentration and the contribution of copper(II) complexes to the total peptide concentration, assuming any set of complex equilibria $\mathbf{M}_{p} \mathrm{H}_{q} \mathrm{~A}_{r}$. Equations were derived according to the approach indicated by Sillén. ${ }^{1}$ The formula of the main complexes and prelim. inary values of corresponding equilibrium constants were evaluated graphically. These constants were refined by a generalized least square method, ${ }^{2}$ using the high speed computer, SAAB D21, and the Letagrop program. ${ }^{3}$
\end{abstract}

Q

uantitative studies on copper(II) complexes of peptides have been made by several investigators. ${ }^{4-23}$ Dobbie and Kermack ${ }^{7}$ investigated some dipeptides by titration with sodium hydroxide using a glass electrode. The data obtained for glycylglycine were explained by the formation of five $\mathrm{MH}_{q} \mathrm{~A}_{r}$ species: $(1,0,1),(1,-1,1),(1,-2,1),(1,-1,2),(1,-2,2)$. The complex $\mathrm{MH}_{1} \mathrm{~A}$ was supposed to be formed by dissociation of a proton from the peptide bond nitrogen, which thereby became coordinated to copper(II), (cf. Refs. 24,25). Rabin et al. ${ }^{10,14}$ made similar measurements on a number of substituted glycine compounds. They were able to confirm the results of Dobbie and Kermack ${ }^{7,8}$ and furthermore the transformation of $\mathrm{MA}$ into $\mathrm{MH}_{-1} \mathrm{~A}$ was found to be accompanied by a shift of the copper(II) coordination from the oxygen to the nitrogen of the peptide bond. ${ }^{10,11,13,14}$ The infrared investigations of Rosenberg ${ }^{26}$ indicate that this copper(II) coordination also occurs in the solid state. Strandberg et al. ${ }^{19}$ came to the same conclusion by $\mathrm{X}$-ray diffraction analysis of $\mathrm{Cu}(\mathrm{II})$-glycylglycine crystals. 
A crystallized complex of glycylglycylglycine ${ }^{20}$ also involves the amide nitrogen as dentate group, but the cupric ion is bound in dimeric form. Such a complex has not been detected in solution, ${ }^{8,12,14,21}$ though a dinuclear glycylglycine species has been reported..$^{18,21,23}$ On reinvestigation of the glycylglycine system ${ }^{23}$ it was also found that the measurements were explained better by one $\mathrm{MH}_{q} \mathrm{~A}_{2},(1,-1,2)$, than by two such species (cf. Refs. 15, 18).

Although most investigators agree that copper(II) induces proton dissociation from the peptide bond, ${ }^{7-23}$ there are differences of opinion regarding the number of species and their composition. It must be kept in mind that many of the conclusions drawn about the equilibria have been based on data from only one titration. Furthermore, the electrometric measurements have been made almost exclusively with glass electrodes, sometimes in uncontrolled ionic media. It might therefore be worthwhile to investigate copper(II) complexes of some peptides using both the glass electrode and the amalgam electrode. The data obtained should be treated by methods, which do not presuppose the complexes formed. This approach may be particularly suited for peptides, which include some further dentate groups. Moreover, a limited amount of work has been reported for such ligands. Imidazole peptides have been studied by Dobbie and Kermack, ${ }^{7}$ Martin and Edsall, ${ }^{17}$ and Doran et al. ${ }^{22}$ This investigation deals with the copper(II) complexes of $\mathrm{O}$-phosphorylserylglutamic acid. It is a part of a systematic study ${ }^{27-29}$, intended to contribute to the understanding of such metal-ion-dependent enzyme reactions that involve phosphorylated substrate or phosphorylated enzyme.

\section{SYMBOLS}

A

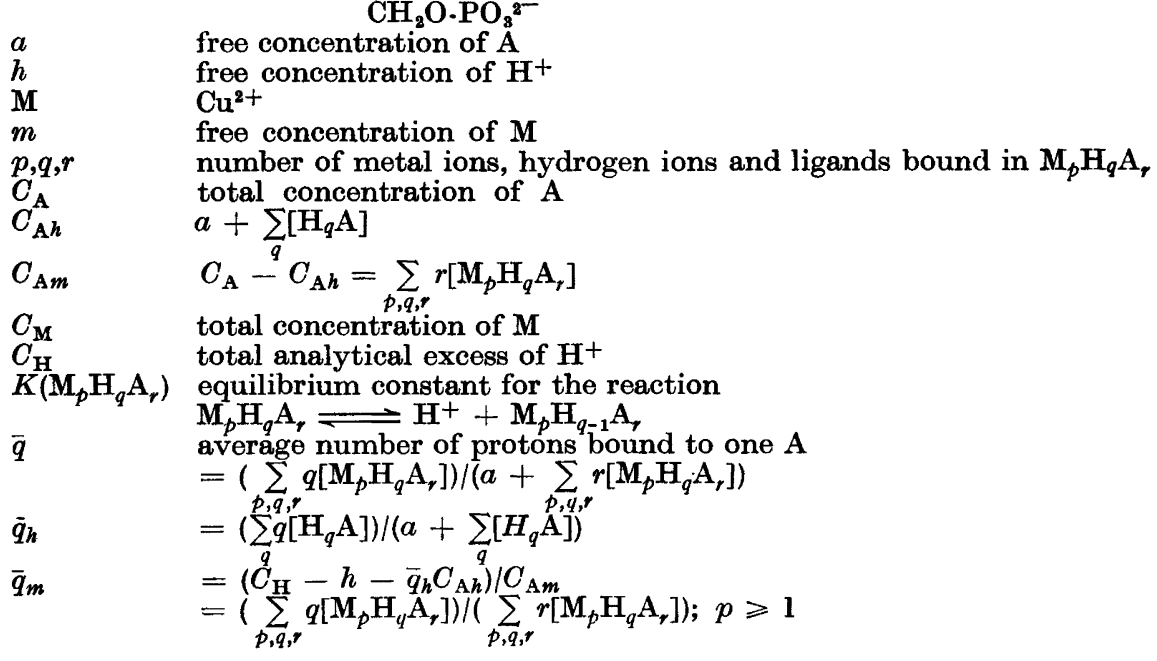

free concentration of $\mathrm{A}$

$\mathrm{Cu}^{2+}$

number of metal ions, hydrogen ions and ligands bound in $\mathrm{M}_{p} \mathrm{H}_{q} \mathrm{~A}_{\text {, }}$ total concentration of $\mathrm{A}$

$a+\sum_{q}\left[\mathrm{H}_{q} \mathrm{~A}\right]$

$C_{\mathrm{A}}-C_{\mathrm{A} h}=\sum_{p, q, r} r\left[\mathrm{M}_{p} \mathrm{H}_{q} \mathrm{~A}_{r}\right]$

total analytical excess of $\mathrm{H}^{+}$

equilibrium constant for the reaction

average number of protons bound to one $A$

$=\left(\sum_{p, q, r} q\left[\mathrm{M}_{p} \mathrm{H}_{q} \mathrm{~A}_{r}\right]\right) /\left(a+\sum_{p, q, r} r\left[\mathrm{M}_{p} \mathrm{H}_{q} \mathrm{~A}_{r}\right]\right)$

$=\left(\sum_{q}^{p, q, r}\left[\mathrm{H}_{q} \mathrm{~A}\right]\right) /\left(a+\sum_{q}\left[H_{q} \mathrm{~A}\right]\right)$

$=\left(C_{\mathrm{H}}-h-\bar{q}_{h} C_{\mathrm{A} h}\right) / C_{\mathrm{A} m}$

$=\left(\sum_{p, q, r}^{\mathrm{H}} q\left[\mathrm{M}_{p} \overline{\mathrm{H}}_{q} \mathrm{~A}_{r}\right]\right) /\left(\sum_{p, q, r} r\left[\mathrm{M}_{p} \mathrm{H}_{q} \mathrm{~A}_{r}\right]\right) ; p \geqslant 1$

conjugate base of $\mathrm{O}$-phosphorylserylglutamic acid,<smiles>NC(CO[N+](=O)[O-])C(CC(=O)[O-])C(=O)[O-]</smiles>

Acta Chem. Scand. 19 (1965) No. 6 


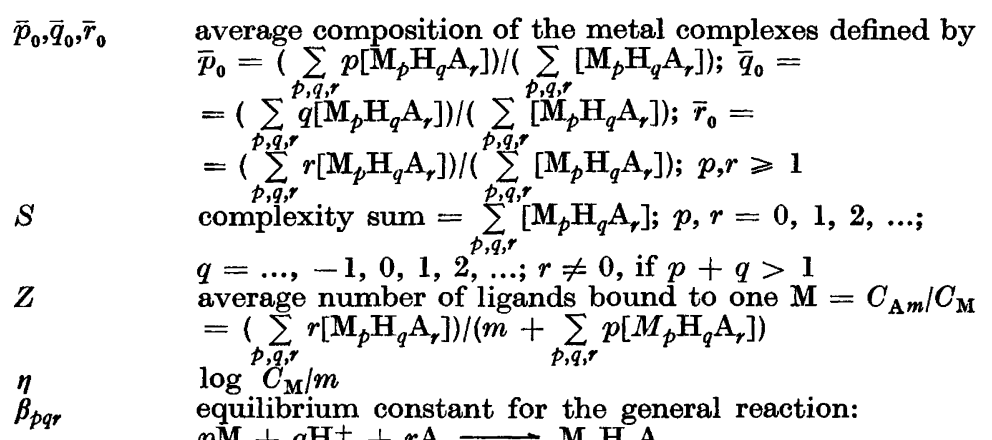

$p \mathbf{M}+q \mathbf{H}^{+}+r \mathbf{A} \rightleftharpoons \mathbf{M}_{p} \mathbf{H}_{q} \mathbf{A}_{r}$

Species with proton deficit (formed by hydrolysis or by displacement of a proton from the peptide bond) are designated negative $q$. $\beta_{p q r}$ equals unity, when two of the integers, $p, q, r$, are zero.

All charges are omitted for the sake of convenience.

\section{METHOD}

The experiments have been carried out as potentiometric titrations. The analytical hydrogen ion concentration, $C_{\mathrm{H}}$, of copper(II)-phosphorylserylglutamic acid solutions with $\mathrm{K}\left(\mathrm{NO}_{3}\right)$ as the ionic medium was varied by adding $\mathrm{KOH}$ or $\mathrm{HNO}_{3}$, while $C_{\mathrm{M}}$ and $C_{\mathrm{A}}$ were kept constant in each run (cf. Ref. 30). The hydrogen ion concentration and the copper(II) concentration were measured with the cells:

- SE / equilibrium solution / GE +

$E_{g}=E_{g}^{\circ}+59.155 \log h+E_{j}$

- Cu-Hg / equilibrium solution / $\mathrm{SE}+$

$E_{m}=E_{m}^{\circ}-29.58 \log m-E_{j}$

GE denotes a glass electrode and SE the reference half cell:

$150 \mathrm{mM} \mathrm{KNO}_{3} / 150 \mathrm{mM} \mathrm{K}^{+}, 140 \mathrm{mM} \mathrm{NO}_{3}^{-}, 10 \mathrm{mM} \mathrm{Cl}^{-} / \mathrm{AgCl}, \mathrm{Ag}$

In absence of ligand we obtain from eqn. 2

$E_{\mathrm{C}_{\mathrm{M}}}=E_{m}+E_{j}=E_{m}^{\circ}-29.58 \log C_{M}$

Whence

$E_{\mathrm{M}}=E_{m}+E_{j}-E_{C_{\mathrm{M}}}=29.58 \log C_{\mathrm{M}} / m$

We have assumed by regarding eqns. $1-4$ as valid that the activity factors are approximately constant, so that they may be included in $E^{\circ}$. This condition could be fullfilled by keeping the potassium ion concentration constant at $0.15 \mathrm{M}$ in all runs. Since $C_{\mathrm{A}}$ usually was in excess of $C_{\mathrm{M}}$, the interaction between $\mathrm{K}^{+}$and the peptide may dominate over that between $\mathrm{Cu}^{2+}$ and $\mathrm{NO}_{3}^{-}$(cf. Ref. 31).

\section{EXPERIMENTAL}

\section{A p paratus}

The measurements were performed in a paraffin oil thermostat at $25.00 \pm 0.03^{\circ}$, which was kept in a room thermostated at $25.00 \pm 0.5^{\circ}$. The titration vessel, volume $15 \mathrm{ml}$, had a constriction in the lower part that allowed titration of small volumes $(2 \mathrm{ml})$. It had inlets for a capillary for $\mathrm{N}_{2}$, electrodes, mechanical stirrer and two capillaries connected to Agla micrometer syringes. The syringes were mounted on a rack, which could be moved horizontally in two directions as well as vertically with a precision better than $1 \mathrm{~mm}$. Thereby the burette capillaries could easily be dipped down in the titration solution at each delivery of titrant.

Acta Chem. Scand. 19 (1965) No. 6 
Titration vessel, burettes and pipettes were filled with $\mathrm{N}_{2}$ before the solutions were introduced. The solutions were pretreated with $\mathrm{N}_{2}$, and a vigorous stream of $\mathrm{N}_{2}$ was passed through the solution during the course of a titration. The nitrogen was taken from a steel cylinder, and was freed from $\mathrm{O}_{2}$ by hot activated copper ${ }^{32}$ and then led through washing bottles with $10 \% \mathrm{NaOH}, 10 \% \mathrm{H}_{2} \mathrm{SO}_{4}$, and $0.15 \mathrm{M} \mathrm{KNO}_{3}$. A "Wilnelm" salt bridge designed by Forsling, Hietanen, and Sillén ${ }^{33}$ was used. The Ag,AgCl electrodes were made according to Brown. ${ }^{34}$ The glass electrodes (Beckman No. 40498) were checked against a quinhydrone electrode in the $\mathrm{pH}$ region 2 to 6.5 , using a citric acid-phosphate buffer. Some tests were also made using a hydrogen electrode in the range pH 2 to 9.5 , in an acetate-phosphate buffer. The emf's of cell (I) for the glass electrodes selected kept constant within $\pm 0.2 \mathrm{mV}$ relative to those of the quinhydrone or $\mathrm{H}_{2}$ electrode cells.

The emf's of the glass electrode cell was measured by a Radiometer valve potentiometer (PHM 4) to $\pm 0.2 \mathrm{mV}$. It had been calibrated against a Leeds \& Northrup compensator type $K_{2}$. The amalgam emf's (cell II) as well as the quinhydrone and $H_{2}$-electrode emf's were measured to $0.02 \mathrm{mV}$ by the Leeds \& Northrup compensator, using a Honeywell electronic null indicator, type $104 \mathrm{~W} 1-\mathrm{G}$, and a saturated Weston standard cell. All pipettes, burettes and flasks were calibrated, using water. The burettes were also calibrated using mercury.

\section{Experimental details of the emf measurements}

The solutions used in the titrations had the following composition:

$\mathrm{S}_{0}: C_{\mathrm{M}} \mathrm{mM} \mathrm{Cu}^{2+},\left(150-2 C_{\mathrm{M}}-\mathrm{H}_{0}\right) \mathrm{mM} \mathrm{K}^{+}, H_{0} \mathrm{mM} \mathrm{H}^{+}, 150 \mathrm{mM} \mathrm{NO}_{3}^{-}$;

$\mathrm{T}_{0}: 2 C_{\mathrm{M}} \mathrm{mM} \mathrm{Cu}{ }^{2+},\left(300-4 C_{\mathrm{M}}-2 \mathrm{H}_{0}\right) \mathrm{mM} \mathrm{K}^{+}, 2 \mathrm{H}_{0} \mathrm{mM} \mathrm{H}^{+}, 300 \mathrm{mM} \mathrm{NO}_{3}^{-}$;

$\mathrm{S}_{1 \mathrm{~A}}: C_{\mathrm{ML}} \mathrm{mM} \mathrm{Cu}^{2+}, 2 C_{\mathrm{A}} \mathrm{mM}$ ligand, $h_{m} \mathrm{mM} \mathrm{H}^{+}, 150 \mathrm{mM} \mathrm{K}^{+},\left(150+2 C_{\mathrm{M}}+h_{m}{ }^{3}\right) \mathrm{mM} \mathrm{NO}_{3}^{-}$;

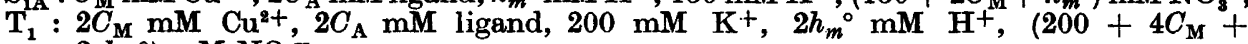
$\left.2 h_{m}^{\circ}\right) \mathrm{mM} \mathrm{NO}_{3}^{-}$;

$\mathrm{T}_{2}: 2 C_{\mathrm{M}} \mathrm{mM} \mathrm{Cu} \mathrm{Cu}^{2+}, 2 h_{m}^{\circ} \mathrm{mM} \mathrm{H} \mathrm{H}^{+},\left(100-2 C_{\mathrm{M}}-2 h_{m}{ }^{\circ}\right) \mathrm{mM} \mathrm{K}+, 100 \mathrm{mM} \mathrm{NO}_{3}^{-}$.

The quantity, $h_{m}^{\circ},(<0.1 \mathrm{mM})$, denotes excess of $\mathrm{H}^{+}$obtained from the acidified copper(II) stock solution.

The liquid junction potential, $E_{j}$, was determined by the procedure described by Biedermann and Sillén. ${ }^{31}$ Titrations were carried out in absence of peptide at constant $C_{\mathrm{M}}$ and constant anion concentration $\left(0.15 \mathrm{M} \mathrm{NO}_{3}^{-}\right)$. A starting solution, $\mathrm{S}_{0}$, was titrated with equal amounts of $0.1 \mathrm{M} \mathrm{KOH}$ and $\mathrm{T}_{0}$ to $-\log h=3$. Equal amounts of $0.2 \mathrm{M}$ $\mathrm{HNO}_{3}$ and $\mathrm{T}_{2}$ were then added to $-\log h=1.5$. Glass electrode and amalgam emf's were recorded after each addition. From the first part of the titration the excess of hydrogen ions, $H_{0}$, in $\mathrm{S}_{0}$ was determined by a Gran plot ${ }^{35}$ using approximate values of $E_{j}$. Hydrolysis was neglected. ${ }^{36,87}$ From the second part the quantities, $E_{g}-59.15 \log h$ and $E_{m}+29.58 \log C_{\mathrm{M}}$, of eqns. 1 and 2 were calculated and plotted against $h$. These plots were linear up to - $\log h=1.7$ with the intercepts $E_{g}^{\circ}=163.8 \mathrm{mV}$ and $E_{m}{ }^{\circ}=106.36 \mathrm{mV}$ ( $h$ and $C_{M}$ in eqns. 1 and 2 in $\mathrm{mM}$ ) and approximately the same slope $=-(312 \pm 20) 10^{-3}$. The $E^{\circ}$ values agree to $\pm 0.1 \mathrm{mV}$ and $\pm 0.05 \mathrm{mV}$ with those finally computed from the first part of the titration, using $E_{j}=-312 h(h$ in M).

The latter procedure was also used to calculate $E_{g}^{\circ}$ and $E_{C_{M}}$ from the first part of each separate run. In this part $\mathrm{S}_{0}$ was titrated with $\mathrm{KOH}$ and $\mathrm{T}_{0}$. The second part started by an addition of $\mathrm{S}_{1 \mathrm{~A}}$ so that the volume of the titration solution was doubled. Then $0.1 \mathrm{M} \mathrm{KOH}$ and $\mathrm{T}_{1}$ were added in equal amounts to $-\log h=9$. Some titrations were interrupted at an earlier point, since stable emf's could not be recorded. $E_{\mathrm{g}}^{\circ}$ remained fairly constant within $0.1 \mathrm{mV}$ from day to day. $E_{C_{\mathrm{M}}}$ varied with about $0.05 \mathrm{mV}$ for titrations with the same $C_{\mathrm{M}}$. The average $E_{C_{\mathrm{M}}}$, plotted against $\log C_{\mathrm{M}}$, obeyed eqn. 3 and gave $E_{m}{ }^{\circ}=106.38 \mathrm{mV}$.

The amalgam emf's generally required $15 \mathrm{~min}$ to attain a constant value, while the glass emf's became constant within a few minutes. The potentials then remained stable for several hours. The time required to reach equilibrium increased somewhat with $-\log h$, and sometimes was up to $1 \mathrm{~h}$ above $\mathrm{pH}$. It was also longer in the first measuring point and at low $C_{\mathrm{M}}$.

At least two titrations were made for each couple $C_{\mathrm{A}}, C_{\mathrm{M}}$, some with different stock solutions. The difference was in general within $0.4 \mathrm{mV}$ in $E_{g}$ and $0.15 \mathrm{mV}$ in $E_{m}$ for 

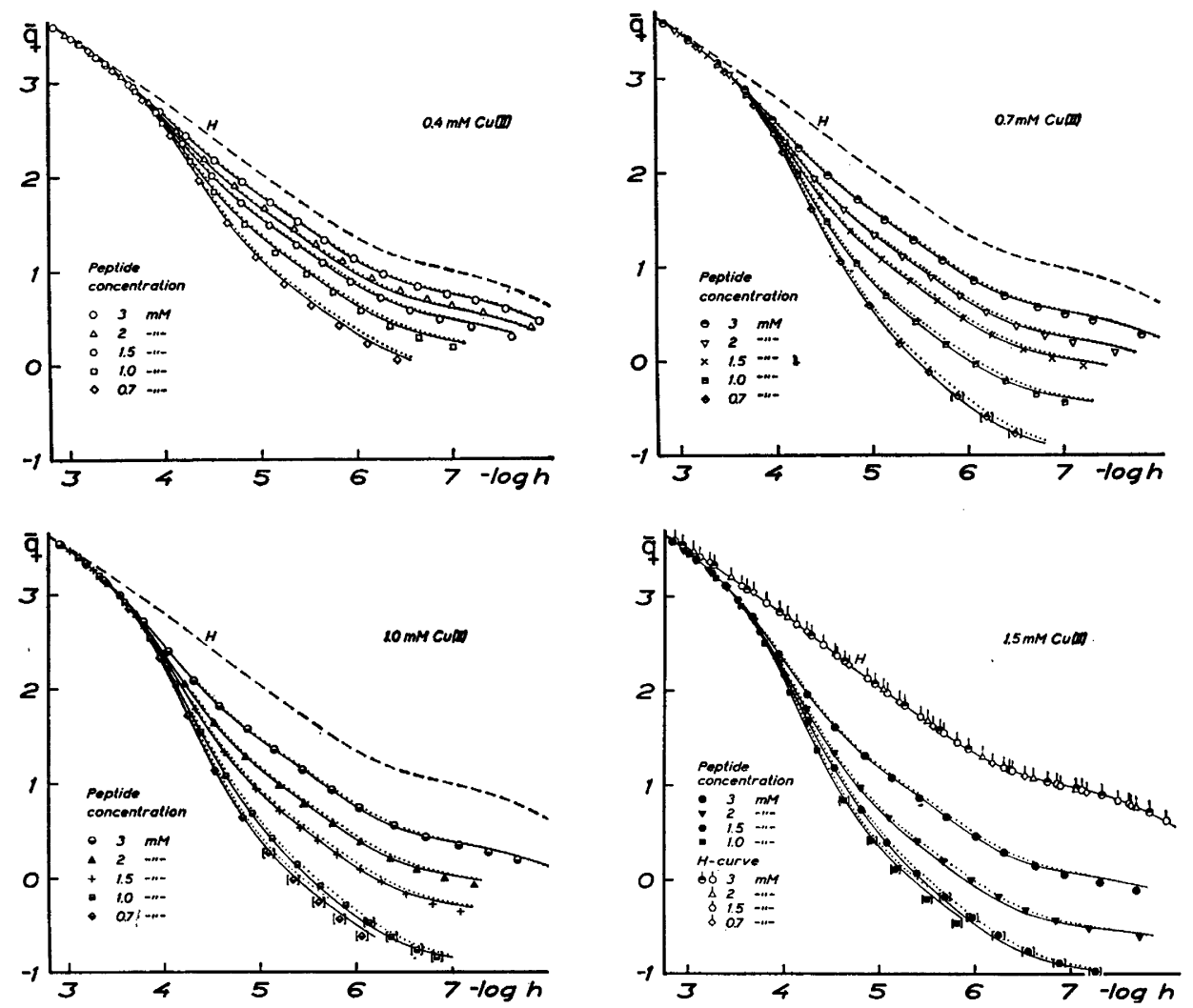

Fig. 1. Copper(II) complexes of O-phosphorylserylglutamic acid in $0.15 \mathrm{M} \mathrm{K}\left(\mathrm{NO}_{3}\right)$

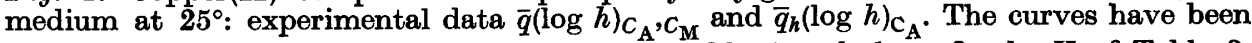
calculated from the equilibrium constants of Table 4 and the refined $\mathrm{p} K$ of Table 3; full drawn curves, set (7); dotted curves, set (2); H-curves (dashed), equilibria in absence of $\mathrm{Cu}(\mathrm{II})$, refined $\mathrm{p} K$ only.

$-\log h<5$. In the higher range there was a somewhat increased spread, especially for the amalgam emf's, which could be reproduced only to $\pm 0.5 \mathrm{mV}$.

When $C_{\mathrm{M}} \geqslant C_{\mathrm{A}}$, the data, $\left(E_{g}, C_{\mathrm{H}}, C_{\mathrm{A}}, C_{\mathrm{M}}\right)$, were checked by titration in absence of amalgam. In some cases a significant deviation was noted before the emf's drifted, or the amalgam was visibly discoloured (cf. Ref. 36). Data beyond this point were rejected.

\section{Materials and analysis}

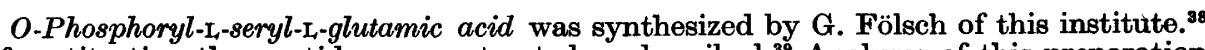
Before titration the peptide was pretreated as described. ${ }^{39}$ Analyses of this preparation have been published previously. ${ }^{39}$ Stock solutions, $4 C_{\mathrm{A}} \mathrm{mM}$, were made for each $C_{\mathrm{A}}$ so that $S_{1 A}$ and $T_{1}$ could be easily prepared. They were standardized by potentiometric titration. Some of the titrations were made at constant $C_{\mathrm{A}}$ according to the procedure described above. In both cases a Gran plot ${ }^{35}$ was used in the following version:

$$
\left(V_{E_{a}}-V\right) 10^{-E_{g} / 59.15}=\text { const. }\left(V-V_{E_{2}}\right)
$$

Acta Chem. Scand. 19 (1965) No. 6 

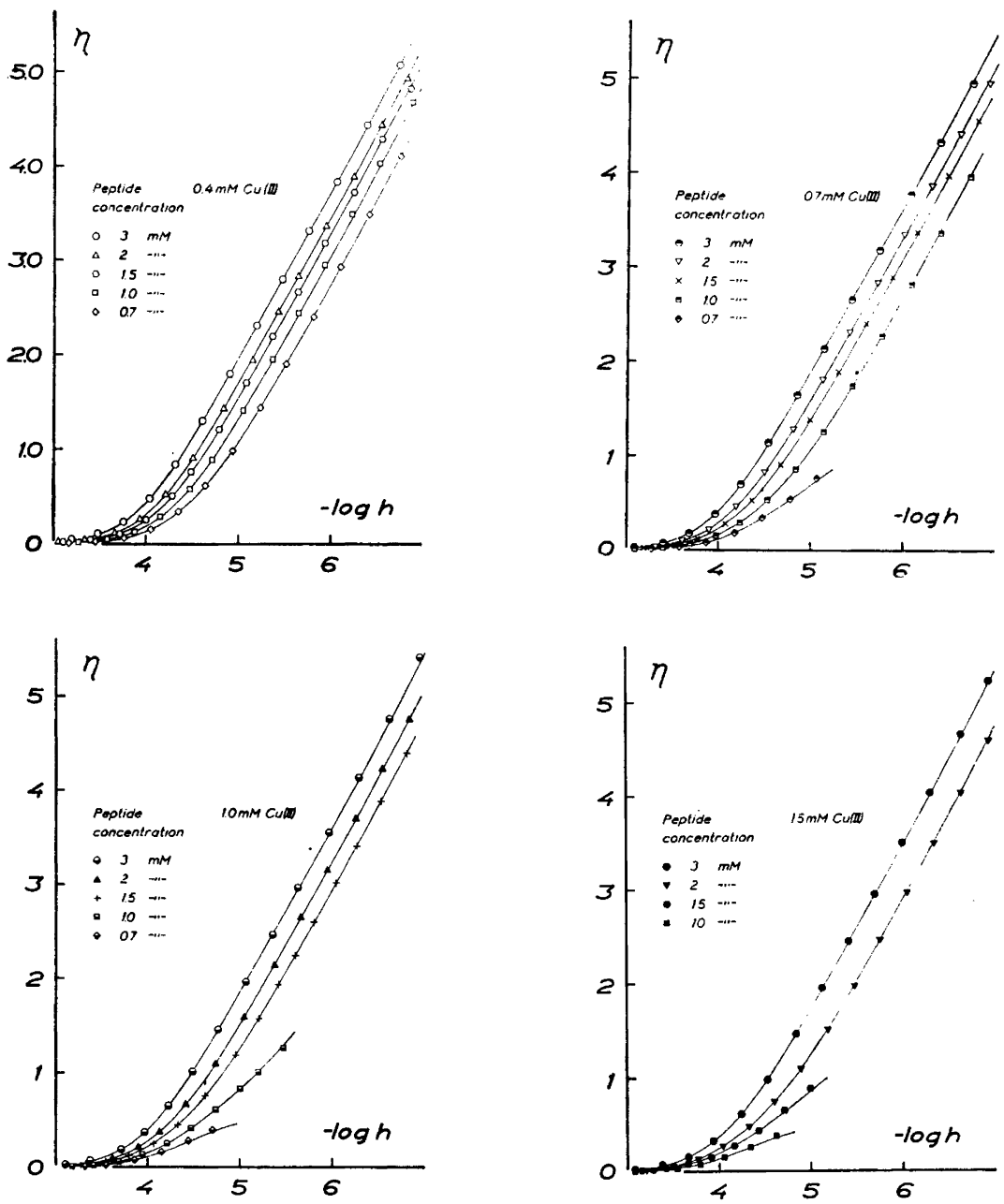

Fig. 2. Copper(II) complexes of O-phosphorylserylglutamic acid in $0.15 \mathrm{M} \mathrm{K}\left(\mathrm{NO}_{3}\right)$ medium: experimental data $\eta(\log h)_{C_{\mathrm{A}}}, C_{\mathrm{M}}$. Drawn curves have been calculated with set (2) of equilibrium constants in Table 4 and the refined $\mathrm{p} K$ of Table 3.

The volume of $\mathrm{KOH}$ consumed at the third equivalence point, $V_{E_{3}}$, is obtained as the abscissa intercept, when the left hand side is plotted against $V$. The quantity $V$ is the volume $\mathrm{KOH}$ added to the titration solution, corrected for $H_{0} . V_{E_{4}}$ is the value of $V$ in the fourth equivalence point. The first value of $V_{E_{4}}$ was found from the titration curve. Then $V_{E_{a}}$ was improved by successive approximation until $V_{E_{a}}$ remained constant.

Potassium nitrate (Merck p.a.) was used without further purification. $\mathrm{Cl}^{-}, \mathrm{Fe}^{3+} \mathrm{qr}$ other heavy metal ions were tested for with $\mathrm{AgNO}_{3}, \mathrm{KSCN}$ and dithizone with negative result. No acidic or basic impurities could be detected in a $3 \mathrm{M}$ solution.

Potassium chloride (Merck p.a., bromide-free). No $\mathrm{Br}^{-}, \mathrm{Fe}^{3+}$, or heavy metal ions could be detected.

Acta Chem. Scand. 19 (1965) No. 6 
Table 1. Copper(II) complexes of O-phosphorylserylglutamic acid at $25^{\circ}$ in $0.15 \mathrm{M} \mathrm{K}\left(\mathrm{NO}_{3}\right)$ medium. Experimental data, $\left(-\log h, \bar{q}_{h}\right)_{\mathrm{A}_{\mathrm{A}}}$, are given, for points used in the Letagrop refinement also $1000\left(q_{\left.h(\text { calc })-\bar{q}_{h}\right)}\right.$ with its sign. For calculation of $\bar{q}_{h \text { (calc) }}$ the refined $\mathrm{p} K$ values listed in Table 3 were used.

$C_{\mathrm{A}}=0.713 m M: 3.216,3.367 ; 3.358,3.264 ; 3.462,3.192 ; 3.564,3.125 ; 3.678,3.046$; $3.781,2.975 ; 3.892,2.897 ; 4.029,2.795 ; 4.137,2.713 ; 4.235,2.631 ; 4.319,2.564 ; 4.401$, $2.493 ; 4.491,2.422 ; 4.577,2.350 ; 4.675,2.276 ; 4.769,2.200 ; 4.874,2.126 ; 4.955,2.063$; $5.040,2.002 ; 5.122,1.941 ; 5.208,1.878 ; 5.291,1.816 ; 5.381,1.753 ; 5.466,1.691 ; 5.554$, $1.628 ; 5.640,1.567 ; 5.737,1.503 ; 5.824,1.440 ; 5.925,1.377 ; 6.002,1.330 ; 6.087,1.284 ;$ $6.177,1.238 ; 6.281,1.190 ; 6.355,1.159 ; 6.430,1.130 ; 6.516,1.097 ; 6.612,1.066 ; 6.726$, $1.065 ; 6.782,1.019 ; 6.971,0.973 ; 7.167,0.926 ; 7.348,0.879 ; 7.632,0.786$;

$C_{\mathrm{A}}=1.45 m M ; 3.036,3.484 ; 3.115,3.422 ; 3.187,3.378,-4 ; 3.266,3.313 ; 3.363,3.249,+1$; $3.461,3.179 ; 3.554,3.113,+4 ; 3.648,3.046 ; 3.729,2.993,+2 ; 3.808,2.935 ; 3.892,2.876,+4 ;$ $3.978,2.812 ; 4.126,2.705,+3 ; 4.209,2.640 ; 4.307,2.564 ; 4.386,2.501 ; 4.467,2.440,+3$; $4.549,2.372 ; 4.631,2.308 ; 4.716,2.243 ; 4.795,2.189, \pm 0 ; 4.873,2.131 ; 5.030,2.015 ; 5.347$, $1.783, \pm 0 ; 5.504,1.667 ; 5.669,1.553 ; 5.810,1.453 ; 5.886,1.403,+2 ; 5.979,1.346 ; 6.094$, $1.283 ; 6.210,1.226,+2 ; 6.296,1.184 ; 6.401,1.142 ; 6.496,1.107 ; 6.582,1.079,+12 ; 6.722$, $1.038 ; 6.891,0.996 ; 6.979,0.975 ; 7.073,0.954,+15 ; 7.208,0.920 ; 7.368,0.878 ; 7.497$, $0.837 ; 7.803,0.714$;

$C_{\mathrm{A}}=1.91 m M ; 2.977,3.521 ; 3.051,3.478 ; 3.119,3.429 ; 3.227,3.352 ; 3.322,3.262 ; 3.443$, $3.201,-7 ; 3.581,3.102 ; 3.680,3.033 ; 3.771,2.969,-4 ; 3.853,2.912 ; 3.934,2.853 ; 4.032$, $2.782,-4 ; 4.117,2.720 ; 4.285,2.592 ; 4.368,2.528 ; 4.449,2.462 ; 4.533,2.397,-6 ; 4.618$, $2.331 ; 4.703,2.264 ; 4.789,2.199 ; 4.878,2.134 ; 4.952,2.079 ; 5.038,2.013 ; 5.143,1.937$; $5.233,1.872 ; 5.321,1.807,-5 ; 5.411,1.742 ; 5.499,1.678 ; 5.588,1.614 ; 5.676,1.550 ; 5.766$, $1.487 ; 5.860,1.423 ; 5.960,1.360 ; 6.073,1.300,-4 ; 6.167,1.246 ; 6.287,1.189 ; 6.397$, $1.143 ; 6.477,1.112 ; 6.603,1.071,+14 ; 6.782,1.020 ; 6.887,0.995 ; 7.045,0.954 ; 7.214$, $0.914 ; 7.367,0.874 ; 7.529,0.824 ; 7.689,0.764 ; 7.856,0.689$;

$C_{\mathrm{A}}=2.97 m M ; 2.857,3.592 ; 3.134,3.412,-1 ; 3.812,2.933,+4 ; 4.160,2.680,+3 ; 4.410$, $2.485,+3 ; 4.741,2.228,+2 ; 5.082,1.976, \pm 0 ; 5.420,1.731,-2 ; 5.671,1.552,-3 ; 5.932$, $1.378,-1 ; 6.134,1.264,+1 ; 6.385,1.152,+4 ; 6.544,1.097,+6 ; 6.702,1.050,+8 ; 6.851$, $1.011,+10 ; 6.931,0.992 ; 7.025,0.971,+10 ; 7.117,0,949 ; 7.208,0.927,+8 ; 7.370,0.885,+4 ;$ $7.615,0.805,-3 ; 7.767,0.742,-7 ; 7.926,0.666,-12 ; 8.005,0.625 ; 8.323,0.440 ; 8.494$, 0.341 ;

$C_{\mathrm{A}}=3.05 \mathrm{mM}: 2.858,3.593 ; 2.937,3.546 ; 3.017,3.491 ; 3.106,3.431 ; 3.182,3.379,-2$; $3.266,3.317 ; 3.358,3.253,+1 ; 3.447,3.190 ; 3.524,3.135,+3 ; 3.606,3.078 ; 3.690,3.020,+2$; $3.767,2.966 ; 3.854,2.904,+3 ; 3.949,2.837 ; 4.029,2.779,+2 ; 4.124,2.708 ; 4.202,2.650$, 士0; $4.286,2.586 ; 4.369,2.521,-1 ; 4.451,2.456 ; 4.542,2.386,-2 ; 4.631,2.315 ; 4.724,2.245,-2$; $4.817,2.175 ; 4.868,2.137,-3 ; 4.949,2.075 ; 5.018,2.025,-2 ; 5.096,1.969 ; 5.180,1.907,-2$; $5.264,1.846 ; 5.357,1.780,-5 ; 5.448,1.713 ; 5.539,1.648,-5 ; 5.629,1.583 ; 5.732,1.513,-7$; $5.825,1.449 ; 5.925,1.386,-5 ; 6.030,1.323 ; 6.134,1.266-1 ; 6.253,1.210 ; 6.354,1.166,+2 ;$ $6.566,1.091,+5 ; 6.653,1.064 ; 6.755,1.037,+8 ; 6.861,1.010 ; 6.963,0.985,+10 ; 7.056$, $0.964 ; 7.147,0.942,+9 ; 7.235,0.921 ; 7.327,0.897,+5 ; 7.498,0.846,+1 ; 7.676,0.781$; $7.825,0.715,-9 ; 7.981,0.638 ; 8.143,0.546 ; 8.316,0.444$;

Copper(II) nitrate (Riedel de Haën p.a.) was recrystallized twice from an acidified water solution by the procedure described by Pedersen. ${ }^{37}$ The dried crystals were found to have a slight deficit of hydrogen ions, 1.3 mmoles per mole $\mathrm{Cu}$ (II). This was determined by potentiometric titration of an acidified copper(II) nitrate solution $(\sim 16 \mathrm{mM})$ with KOH, using a Gran plot. ${ }^{35}$ The nitrate content of the stock solution was analysed by passing the solution through an $\mathrm{H}^{+}$-saturated cation exchanger (Dowex 50W-X2) and titrating for the acid set free. From the difference the copper(II) concentration was calculated. Copper(II) was also analysed by electrolysis. The results of the two methods agreed within $0.2 \%$.

Acta Chem. Scand. 19 (1965) No. 6 
Table 2. Copper(II) complexes of $\mathrm{O}$-phosphorylserylglutamic acid at $25^{\circ}$ in $0.15 \mathrm{M} \mathrm{K}\left(\mathrm{NO}_{3}\right)$ medium. Experimental data (- $\left.\log h, \bar{q}, E_{m}\right)_{C_{\mathrm{A}}}, C_{\mathrm{M}}$ and calculated values on the free ligand concentration (- $\log a_{\mathrm{G}},-\log a_{\mathrm{Cu}}$; from eqns. 10, 21; for every fourth titration) are given, for points used in the Letagrop refinement also $1000\left(\bar{q}_{\text {calc }}-\bar{q}\right), 100\left(E_{m_{\text {calc }}}-\right.$ $\left.E_{m}-E_{j}\right)$ and $1000\left(-\log a_{\mathrm{L}}+\log a\right)$ with their sign; $-\log a_{\mathrm{L}}$ stands for the values obtained by Letagrop. ( $\left(\bar{q}_{\text {calc }},-\log a_{\mathrm{LG}}\right)$ and $\left(E_{m_{\text {calc}}},-\log a_{\mathrm{LCu}}\right)$ were obtained using sets (7) and (2) in Table 4, respectively, and the refined $\mathrm{p} K$ of Table 3.

$$
C_{\mathrm{M}}=0.388 m M
$$

$C_{\mathrm{A}}=0.707 m M, E_{C_{\mathrm{M}}}=118.49 \mathrm{mV} ;-\log h, \bar{q}, 10^{3}\left(\bar{q}_{\mathrm{calc}}-\bar{q}\right),-\log a_{\mathrm{G}}, 10^{3}\left(-\log a_{\mathrm{L}, \mathrm{G}}+\right.$ $\left.+\log a_{\mathrm{G}}\right), E_{m}, 100\left(E_{m}\right.$ (calc) $\left.-E_{m}-E_{j}\right),-\log a_{\mathrm{Cu}}, 10^{3}\left(-\log a_{\mathrm{LCu}}+\log a_{C_{\mathrm{u}}}\right)$ :

$3.207,3.344,12.072,119.01,12.073 ; 3.291,3.275,11.794,119.05,11.795 ; 3.373,3.211$, $11.528,119.13,11.529 ; 3.457,3.129,+8,11.260,-7,119.27, \pm 0,11.262,-9 ; 3.572,3.024$ $10.905,119.54,10.907 ; 3.670,2.933,10.611,119.90,10.612 ; 3.768,2.826,10.326,120.43$, $10.326 ; 3.864,2.708,10.057,121.14,10.056 ; 3.958,2.580,+15,9.803,-5,122.04,+4$ $9.803,-8 ; 4.055,2.446,9.551,123.15,9.551 ; 4.162,2.276,9.285,124.84,9.282 ; 4.251$, $2.131,9.072,126.48,9.070 ; 4.354,1.969,8.835,128.60,8.834 ; 4.448,1.820,+20,8.627, \pm 0$, $130.89,+8,8.627,-8 ; 4.544,1.669,8.421,133.57,8.433 ; 4.648,1.518,8.206,136.78$, $8.230 ; 4.749,1.381,8.003,140.25,8.029 ; 4.850,1.259,7.806,143.86,7.821 ; 4.941$, $1.153,+31,7.634,-5,147.61,+39,7.637,-16 ; 5.042,1.046,7.447,151.88,7.438 ; 5.138$, $0.954,7.275,156.17,7.254 ; 5.238,0.862,7.093,160.95,7.055 ; 5.325,0.786,6.950,165.34$, $6.907 ; 5.423,0.709,+42,6.787,-35,169.98,+65,6.735,+12 ; 5.518,0.632,6.634,174.89$, $6.574 ; 5.619,0.556,6.477,180.00,6.411 ; 5.726,0.479,6.304,185.29,6.245 ; 5.805,0.418$, $+41,6.202,-52,189.54,+49,6.128,+17 ; 5.918,0.341,6.045,195.34,5.946 ; 6.006$, $0.280,5.93,200.0,5.85 ; 6.100,0.219,5.81,205.2,5.73 ; 6.204,0.158,5.68,210.6,5.66 ;$ $6.312,0.097,5.56,216.45,5.47 ; 6.409,0.051,5.44,221.4,5.35 ; 6.505,0.006,5.34,226.7$, $5.25 ; 6.614,-0.040,5.22,232.8,5.13 ; 6.748,-0.085,5.08,239.9,4.99 ; 6.842,-0.116$, $245.0 ; 6.951,-0.146,250.8$;

$C_{\mathrm{A}}=1.00_{8} m M, E_{C_{\mathrm{M}}}=118.62 m V: 3.118,3.406,119.21 ; 3.193,3.357,119.26 ;$ $3.274,3.285,119.34 ; 3.380,3.210,-7,119.51,-4 ; 3.481,3.118,119.78 ; 3.583,3.023$, $120.18 ; 3.693,2.918,120.74 ; 3.791,2.809,+5,121.54,+5 ; 3.879,2.700,122.48 ; 3.975$, $2.578,123.76 ; 4.074,2.449,125.33 ; 4.161,2.320,+26,127.22,-8 ; 4.267,2.176,129.69$; $4.373,2.034,132.64 ; 4.475,1.899,135.94 ; 4.512,1.847,+28,137.34,-16 ; 4.619$, 1.722, $141.24 ; 4.716,1.618,145.03 ; 4.827,1.503,149.78 ; 4.946,1.387,+31,155.36,+10 ; 5.050$ $1.293,160.56 ; 5.147,1.199,165.61 ; 5.252,1.125,170.91 ; 5.368,1.042,+38,176.41,-2$; $5.471,0.968,181.43 ; 5.569,0.895,186.51 ; 5.647,0.833,191.05 ; 5.742,0.770,+31$, $195.52,+4 ; 5.827,0.697,200.41 ; 5.931,0.635,206.06 ; 6.033,0.573,211.33 ; 6.139,0.511$, $+32,216.87,-62 ; 6.222,0.459,221.79 ; 6.334,0.408,227.36 ; 6.437,0.367,232.7 ; 6.523$, $0.326,23 \% .6 ; 6.637,0.285,243.7 ; 6.758,0.244,250.4 ; 6.879,0.213,256.9 ; 6.995,0.183$, $263.5 ; 7.260,0.122,278.7$;

$C_{\mathrm{A}}=1.45 \mathrm{mM} . E_{C_{\mathrm{M}}}=118.58 \mathrm{mV}: 3.024,3.467,119.29 ; 3.107,3.413,119.35 ; 3.197$, $3.348,119.40 ; 3.293,3.271,+3,119.58,-10 ; 3.402,3.186,119.85 ; 3.516,3.088,120.34$; $3.623,2.990,+1,121.00,+1 ; 3.721,2.897,121.84 ; 3.814,2.797,122.94 ; 3.909,2.693,+3$, $124.3 \%,+1 ; 4.003,2.585,126.07 ; 4.094,2.476,128.1 \% ; 4.185,2.364,+7,130.61,-8$; $4.283,2.245,133.72 ; 4.391,2.125,137.42 ; 4.488,2.019,+7,141.23,-24 ; 4.592,1.913$, $145.58 ; 4.697,1.814,150.20 ; 4.792,1.729,+5,154.60,-11 ; 4.892,1.644,159.39 ; 4.989$, $1.567,164.14 ; 5.087,1.489,+7,169.10, \pm 0 ; 5.179,1.419,173.88 ; 5.272,1.349,178.64 ;$ $5.368,1.278,+10,183.61,-14 ; 5.456,1.215,188.06 ; 5.541,1.153,192.4_{9} ; 5.641,1.083$, $+4,197.4_{5},+3 ; 5.742,1.014,202.4_{6} ; 5.824,0.952,207.2_{8} ; 5.928,0.883,+4,212.5_{6},-27$; $6.027,0.821,217.7_{5} ; 6.133,0.760,223.1_{5} ; 6.241,0.705,+3,228.6,+18 ; 6.335,0.658$, $233.7 ; 6.443,0.611,239.7 ; 6.547,0.570,+17,245.2_{5},+16 ; 6.643,0.536,250.4 ; 6.756$, $0.503,256.1 ; 6.853,0.476,+30,261.4,+100 ; 6.956,0.449,266.6 ; 7.188,0.396,278.7$; $7.463,0.329,292.2 ; 7.610,0.290,297.4$

$C_{\mathrm{A}}=1.90_{6} m M, E_{C_{\mathrm{M}}}=118.55 \mathrm{mV}: 2.964,3.509,119.36 ; 3.048,3.462,119.40$; $3.148,3.389,-3,119.50,-16 ; 3.246,3.309,119.68 ; 3.341,3.239,119.94 ; 3.443,3.154,-2$, 
$120.36,-5 ; 3.552,3.065,121.01 ; 3.664,2.968,121.98 ; 3.752,2.883,-10,123.02,+13 ;$ $3.838,2.794,124.36 ; 3.934,2.693,126.40 ; 4.031,2.591,-9,128.45,+11 ; 4.121,2.492$, $131.00 ; 4.216,2.393,133.98 ; 4.309,2.293,-9,137.47,-10 ; 4.411,2.194,141.44 ; 4.509$, $2.099,145.64 ; 4.620,1.999,-18,150.70,-8 ; 4.725,1.910,155.64 ; 4.837,1.821,160.99$; $4.935,1.743,-22,165.95,+13 ; 5.039,1.665,171.14 ; 5.144,1.588,176.51 ; 5.241,1.516,-21$, $181.61,+17 ; 5.344,1.444,186.76 ; 5.428,1.383,191.20 ; 5.501,1.332,-27,194.91,+23$; $5.570,1.281,198.53 ; 5.640,1.230,202.27 ; 5.739,1.165,207.19 ; 5.843,1.095,-35,212.66$, $+4 ; 5.944,1.030,217.96 ; 6.044,0.971,223.1 ; ; 6.148,0.912,-38,228.7_{1},-7 ; 6.236$, $0.867,233.3_{2} ; 6.341,0.819,238.8_{9} ; 6.448,0.775,-33,244.6_{7},+15 ; 6.541,0.741,249.7$; $6.643,0.707,255.7 ; 6.727,0.683,-25,259.6,+70 ; 6.906,0.635,269.1 ; 6.984,0.616$, $273.3 ; 7.091,0.592,279.0 ; 7.317,0.539,289.7 ; 7.645,0.449,306.0 ; 7.818,0.388,312.5$

$C_{\mathrm{A}}=3.05_{5} m M, E_{C_{\mathrm{M}}}=118.55 m V: 2.845,3.589,119.63 ; 2.915,3.542,119.65 ;$ $3.009,3.483,119.72 ; 3.107,3.414,11.774,119.85,11.775 ; 3.197,3.348,+1,11.469,-8$, $120.08,-25,11.471,-10 ; 3.290,3.277,11.162,120.43,11.162 ; 3.387,3.200,10.847$, $120.95,10.847 ; 3.485,3.119,+1,10.537,-9,121.67,-8,10.538,-10 ; 3.577,3.039$, $10.252,122.67,10.253 ; 3.671,2.958,9.968,123.89,9.969 ; 3.762,2.874,+5,9.701,-10$, $125.51,+2,9.701,-11 ; 3.855,2.788,9.434,127.50,9.435 ; 3.947,2.702,9.176,129.91$, $9.178 ; 4.039,2.616,+5,8.924,-13,132.71,+2,8.925,-15 ; 4.129,2.529,8.682,135.95$, $8.680 ; 4.222,2.442,8.437,139.60,8.436 ; 4.318,2.356,+5,8.191,-11,143.59,-18,8.190$, $-12 ; 4.416,2.270,7.946,147.95,7.946 ; 4.517,2.185,7.703,152,66,7.703 ; 4.610,2.106, \pm 0$, $7.484,-9,157.26,-13,7.484,-11 ; 4.709,2.028,7.259,162.06,7.25 \% ; 4.806,1.953$, $7.047,166.88,7.045 ; 4.903,1.879,-3,6.839,-9,171.83,+32,6.838,-11 ; 4.998,1.805$, $6.642,176.89,6.642 ; 5.098,1.733,6.442,182.00,6.443 ; 5.193,1.663,+3,6.259,-7$, $187.00,+28,6.259,-10 ; 5.287,1.595,6.084,191.83,6.084 ; 5.383,1.527,5.912,196.54$, $5.912 ; 5.474,1.459,+4,5.755,-7,201.40,+34,5.754,-9 ; 5.568,1.392,5.599,206.32$, $5.601 ; 5.660,1.326,5.452,211.16,5.454 ; 5.758,1.255,5.303,216.43,5.304 ; 5.862,1.187, \pm 0$, $5.152,-7,221.80,-26,5.153,-11 ; 5.960,1.126,5.015,226.80,5.018 ; 6.061,1.067,4.88$, $231.9_{1}, 4.88 ; 6.162,1.012,-3,4.75_{3},-6,237.4_{3},-21,4.75_{4},-12 ; 6.270,0.960,4.62$, $243.1_{1}, 4.62 ; 6.385,0.908,4.49,249.6_{1}, 4.49 ; 6.515,0.859,+5,4.34_{4}-7,256.4_{1},-1$, $4.33,-12 ; 6.631,0.821,4.22,262.34 .21 ; 6.737,0.791,+11,4.10,-12,268.5,+25$, $4.10_{2},-13 ; 6.833,0.765,4.01,273.3,4.00 ; 6.936,0.740,3.91,279.0,3.900 ; 7.030,0.717$, $3.82,284.9,3.81 ; 7.126,0.695,289.5 ; 7.234,0.670,294 ; 7.552,0.584,312 ; 7.903,0.450,328$;

$$
C_{\mathrm{A}}=0.705 \mathrm{mM}, E_{C_{\mathrm{M}}}=110.36 \mathrm{mV}: \mathbf{3 . 1 7 7 , 3 . 3}
$$

$C_{\mathrm{A}}=0.705 m M, E_{C_{\mathrm{M}}}=110.36 m V: 3.177,3.339,110.84 ; 3.276,3.256,110.84$; $3.374,3.168,+9,110.95,+3 ; 3.476,3.072,111.11 ; 3.582,2.955,111.39 ; 3.688,2.833$, $111.74 ; 3.773,2.718,112.15 ; 3.871,2.567,+5,112.80+10 ; 3.974,2.392,113.65 ; 4.075$ $2.210,114.66 ; 4.179,2.007,115.93 ; 4.276,1.815,117.26 ; 4.375,1.621,-2,118.81,+1 ;$ $4.478,1.426,120.55 ; 4.578,1.229,122.45 ; 4.671,1.062,124.27 ; 4.780,0.880,126.49$; $4.875,0.728, \pm 0,128.59,-41 ; 4.967,0.591,130.66 ; 5.067,0.455,132.93 ; 5.184,0.303$, $135.65 ; 5.286,0.182,138.05 ; 5.389,0.076,140.46 ; 5.477,-0.014,142.40 ; 5.586,-0.119$, $145.0 ; 5.687,-0.210,147.1 ; 5.791,-0.300,149.4 ;(5.883,-0.376,151.4 ; 5.982,-0.450$, $153.6 ; 6.081,-0.525,155.4 ; 6.189,-0.600,157.3 ; 6.280,-0.660,159.3 ; 6.376,-0.719$, $162.3 ; 6.489,-0.779,168.7 ; 6.666,-0.868,180 ;)$

$C_{\mathrm{A}}=1.00_{8} m M, E_{C_{\mathrm{M}}}=110.27 m V: 3.101,3.404,110.85 ; 3.210,3.324,110.88 ; 3.302$, $3.239,+4,110.99,-3 ; 3.400,3.149,111.16 ; 3.506,3.047,111.43 ; 3.601,2.944,111.80$; $3.698,2.832,-1,112.31,+8 ; 3.784,2.718,112.92 ; 3.878,2.578,113.75 ; 3.978,2.424$, $114.89 ; 4.072,2.264,+3,116.23,+7 ; 4.127,2.173,117.05,4.234,1.990,118.95 ; 4.341$, $1.805,121.20 ; 4.444,1.627,-11,123.65,+9 ; 4.532,1.482,126.00 ; 4.634,1.327,128.89$; $4.741,1.171,132.30 ; 4.841,1.037,-10,135.72,+7 ; 4.934,0.923,139.11 ; 5.042,0.799$, $143.41 ; 5.142,0.696,147.50 ; 5.247,0.594,+3,152.29,+26 ; 5.353,0.502,157.22 ; 5.453$, $0.420,161.92 ; 5.556,0.339,-1,167.0,+45 ; 5.679,0.247,172.8 ; 5.766,0.176,17 \% .5$; $5.868,0.105,182.6 ; 5.969,0.034,187.8 ; 6.077,-0.036,193.6 ; 6.176,-0.097,198.6$; $6.278,-0.157,204.3 ; 6.389,-0.217,209.8 ; 6.492,-0.267,215.7 ; 6.608,-0.317,222.0$; $6.712,-0.357,227.7 ; 6.801,-0.387,232.5 ; 6.898,-0.416,237.6 ; 7.009,-0.446,243.3$; 
$C_{\mathrm{A}}=1.45_{4} m M, E_{C_{\mathrm{M}}}=110.39 m V: 3.013,3.473,111.07 ; 3.090,3.414,111.10$; $3.194,3.335,111.19 ; 3.298,3.248,111.35 ; 3.410,3.149,-3 ; 111.64,-1 ; 3.484,3.079$, $111.89 ; 3.586,2.975,112.40 ; 3.687,2.862,-8 ; 113.12,+12 ; 3.778,2.749,114.00 ; 3.874$, $2.616,115.21 ; 3.976,2.467,+1,116.80+7 ; 4.080,2.316,118.75 ; 4.172,2.176,120.84$; $4.266,2.037,-6,123.25, \pm 0 ; 4.363,1.897,126.05 ; 4.466,1.757,129.35 ; 4.568,1.624,-10$, $133.11,-8 ; 4.673,1.499,137.23 ; 4,774,1.388,141.55 ; 4.873,1.292,-11,145.85,-4$; $4.991,1.182,151.41 ; 5.101,1.086,156.74 ; 5.201,1.003,-2,161.8,-19 ; 5.296,0.928$, $166.55 ; 5.399,0.854,171.74 ; 5.497,0.779,-3,176.85,-21 ; 5.591,0.711,181.65 ; 5.683$, $0.644,186.46 ; 5.770,0.577,-5,191.30,-62 ; 5.871,0.510,196.19 ; 5.949,0.457,200.36$; $6.031,0.404,-12,204.7_{1},-47 ; 6.132,0.339,210.1_{5} ; 6.253,0.273,216.4 ; 6.354,0.221,-3$, $221.9_{3}-52 ; 6.468,0.168,228.0_{5} ; 6.579,0.123,234.1_{5} ; 6.685,0.084,+18,239.9,-38$; $6.788,0.051,245.3_{5} ; 6.878,0.025,250.4 ; 6.978,-0.001,256 .{ }_{1} ; 7.091,-0.026,262 .{ }_{2}$; $7.208,-0.052,268_{{ }_{4}} ; 7.398,-0.091,278_{8}$;

$C_{\mathrm{A}}=1.91_{3} m M, E_{C_{\mathrm{M}}}=110.42 m V: 2.951,3.515,111.24 ; 3.019,3.473,111.26$; $3.105,3.406,11.987,111.29,11.989$; 3.216, 3.319,-1, 11.613,-7, 111.49,-15, 11.615, -9; $3.324,3.230,11.260,111.71,11.260 ; 3.425,3.136,10.937,112.13,10.939 ; 3.522,3.044,-4$, $10.635,-5,112.62,+1,10.638,-9 ; 3.615,2.944,10.354,113.35,10.35 \% ; 3.709,2.840$, $10.079,114.24$. 10.080; 3.807, 2.722, - 1, 9.801,-4, 115.50, $9,9.802,-7 ; 3.899,2.600$, $9.550,117.00,9.549 ; 3.999,2.466,9.286,119.05,9.287 ; 4.098,2.332,+7,9.033,-9$, $121.37,+2,9.032,-13 ; 4.198,2.197,8.786,124.21,8.780 ; 4.303,2.062,8.534,127.54$, $8.526 ; 4.404,1.938,+4,8.296,-16,131.15,-11,8.285,-11 ; 4.502,1.825,8.069,135.05$, $8.058 ; 4.608,1.712,7.830,139.40,7.818 ; 4.710,1.610,+2,7.603,-12,144.01,-13$, $7.591,-6 ; 4.810,1.519,7.387,148.55,7.379 ; 4.926,1.418,7.141,154.17,7.135 ; 5.022$, $1.338,6.944,159.09,6.943 ; 5.124,1.258,+9,6.742,+2,164.15,-5,6.743,-5 ; 5.226$, $1.178,6.548,169.39,6.549 ; 5.319,1.109,6.378,174.16,6.379 ; 5.417,1.040,+10,6.204,+5$, $179.08,+1,6.206,-4 ; 5.510,0.971,6.046,183.94,6.048 ; 5.616,0.893,5.872,189.54$, $5.876 ; 5.713,0.825,5.721,194.48,5.726 ; 5.809,0.757,+5,5.578,+6,199.55,-29$, $5.582,-5 ; \quad 5.911, \quad 0.690,5.433,204.92,5.437 ; 5.997, \quad 0.633,5.315,209.5,5.321$; $6.094,0.575,-1,5.188,+8,214.2_{5},-14,5.195,-9 ; 6.187,0.519,5.07,219.1_{6}, 5.08 ; 6.296$, $0.462,4.94,224.9_{6}, 4.95 ; 6.394,0.415,+11,4.82_{5},+11,230.0_{1},+15,4.83_{2},-9 ; 6.504$, $0.369,4.70,235.91,4.71 ; 6.601,0.331,4.60,241.2_{3}, 4.60 ; 6.709,0.294,+29,4.48,+11$, $247.0_{0},+40,4.49,-9 ; 6.804,0.267,4.38,252.0,4.39 ; 6.904,0.239,4.28,25 \% .2,4.29$; $6.997,0.216,4.19,262.1,4.20 ; 7.095,0.193,267.0 ; 7.200,0.170,271.8 ; 7.301,0.147,276.8$; $7.540,0.093,286.1 ; 7.959,-0.034,302$;

$C_{\mathrm{A}}=3.04, m M, E_{C_{\mathrm{M}}}=110.38 m V: 2.838,3.588,111.41 ; 2.921,3.532,111.44 ;$ $3.013,3.470,111.46 ; 3.096,3.410,+4,111.63,-26 ; 3.196,3.332,111.89 ; 3.299$, o.246, $112.22 ; 3.401,3.159,+1,112.78,-10 ; 3.498,3.070,113.54 ; 3.594,2.977,114.53 ; 3.683$, $2.884,+3,115.69,+7 ; 3.785,2.774,117.52 ; 3.877,2.669,119.70 ; 3.970,2.561,+13$, $121.84, \pm 0 ; 4.068,2.453,124.78 ; 4.159,2.355,127.83 ; 4.245,2.263,+8,131.12,-11$ $4.336,2.172,134.73 ; 4.443,2.070,139.30 ; 4.545,1.974,-1,144.14,-9 ; 4.647,1.886$, $149.01 ; 4.752,1.798,154.14 ; 4.854,1.717,-4,159.19,+15 ; 4.945,1.645,163.87 ; 5.039$, $1.573,168.59 ; 5.136,1.500,+5,173.69,+20 ; 5.236,1.427,178.8_{6} ; 5.335,1.355,183.95$; $5.435,1.284,+8,189.1_{1},+21 ; 5.532,1.213,194.0_{6} ; 5.630,1.143,199.1_{0} ; 5.737,1.071$, $204.5_{5} ; 5.844,0.994,+1,210.3_{3},-6 ; 5.935,0.924,216.0_{3} ; 6.066,0.854,221.8_{6} ; 6.158$, $0.803,-3,226.5_{5},+13 ; 6.271,0.745,232.5_{6} ; 6.384,0.692,238.4_{7} ; 6.506,0.642,+9,245.2_{4}$, $+31 ; 6.623,0.603,250.1 ; 6.731,0.568,25 \% .1 ; 6.820,0.544,+23,262.1,+92 ; 6.914,0.519$, $266.8 ; 7.015,0.495,272.4 ; 7.121,0.471,278.1 ; 7.308,0.427,287.4 ; 7.481,0.384,295.2$; $7.660,0.332,302_{.5} ; 7.823,0.276,310.2$;

$$
C_{\mathrm{M}}=0.969 \mathrm{mM}
$$

$C_{\mathrm{A}}=0.707 m M, E_{C_{\mathrm{M}}}=106.83 \mathrm{mV}: 3.183,3.333,107.29 ; 3.239,3.273,107.31 ; 3.317$, $3.202,107.36 ; 3.377,3.154,107.44 ; 3.452,3.078,-2,10 \% .54,+3 ; 3.548,2.974,107.74$; $3.638,2.848,108.01 ; 3.750,2.688,108.47 ; 3.856,2.511,109.07 ; 3.958,2.322,+4$, $109.81,+5 ; 4.056,2.125,110.69 ; 4.153,1.922,111.63 ; 4.250,1.716,112.65 ; 4.347,1.508$, $113.81 ; 4.442,1.314,+3,114.97,-8 ; 4.532,1.134,116.15 ; 4.620,0.968,117.26 ; 4.709$, $0.802,+19,118.45,-37 ; 4.816,0.637,120.1 ; 4.907,0.502,121.1 ;(4.992,0.381,122.0 ; ; 5.087$, $0.261,123.0 ; 5.176,0.156,123.9 ; 5.259,0.066,124.7 ; 5.344,-0.024,125.4 ; 5.439$, 
$-0.114,126.2 ; 5.519,-0.189,126.9 ; 5.608,-0.264,127.6 ; 5.676,-0.323,128.2 ; 5.747$, $-0.383,128.8 ; 5.824,-0.443,129.4 ; 5.903,-0.502,130.3 ; 5.978,-0.562,131.1 ; 6.056$, $-0.622,132.3 ;)$

$C_{\mathrm{A}}=1.00_{8} m M, E_{C_{\mathrm{M}}}=106.84 \mathrm{mV}: 3.109,3.392,107.40 ; 3.182,3.337,107.44 ;$ $3.256,3.268,10 \% .50 ; 3.332,3.198,+1,10 \% .60,-2 ; 3.412,3.119,10 \% .74 ; 3.502,3.023$, $107.99 ; 3.592,2.915,108.34 ; 3.684,2.798,-2,108.78,+4 ; 3.773,2.671,109.36 ; 3.857$, $2.538,110.01 ; 3.948,2.378,110.90 ; 4.039,2.216,-7,111.88,+11 ; 4.125,2.050,113.04$; $4.210,1.882,114.35 ; 4.296,1.713,-2,115.7 \%,+5 ; 4.385,1.544,11 \% .88 ; 4.472,1.385$, $119.05 ; 4.559,1.227,120.89 ; 4.646,1.079,122.79 ; 4.739,0.931,-5,124.91,-13 ; 4.835$, $0.795,12 \% .04 ; 4.918,0.680,129.05 ; 5.002,0.565,131.29 ; 5.062,0.491,-12,132.7 \%,+4$; $5.122,0.418,134.39 ; 5.203,0.325,136.62 ; 5.290,0.231,139.06 ; 5.384,0.138,-17,141.71$, $+33 ; 5.469,0.056,144.15 ; 5548,-0.016,146.40 ; 5.629,-0.088,148.89 ; 5.712,-0.160$, $151.33 ; 5.810,-0.232,153.8_{5} ;\left(5.887,-0.293,156.0_{5} ; 5.965,-0.354,158.2_{5} ; 6.049,-0.415\right.$, $160.6_{5} ; 6.132,-0.476,163.0_{5} ; 6.206,-0.526,165.1_{5} ; 6.282,-0.577,167.2 ; 6.363,-0.627$, $169.4_{5} ; 6.453,-0.678,171.8 ; 6.537,-0.718,174.5 ; 6.631,-0.768,178.5 ; 6.720,-0.798$, $\left.182.5 ; 6.838,-0.848,188_{.3} ;\right)$

$C_{\mathrm{A}}=1.45_{7} m M, E_{C_{\mathrm{M}}}=106.84 m V: 3.020,3.461,10 \% .51 ; 3.101,3.401,12.123$, $10 \% .54,12.125 ; 3.190,3.334,11.822,10 \% .64,11.824 ; 3.271,3.260,-3,11.554,-8,10 \% .74$, $-5,11.556,-10 ; 3.359,3.178,11.271,107.94,11.273 ; 3.451,3.087,10.983,108.22$, $10.985 ; \quad 3.543,2.987,-6,10.702,-8,108.63,+2,10.703,-10 ; 3.631,2.878,10.441$, $109.15,10.442 ; 3.719,2.764,10.187,109.82,10.189 ; 3.803,2.645,-3,9.956,-4,110.64$, $+8,9.956,-7 ; 3.892,2.508,9.719,111.72,9.719 ; 3.977,2.368,9.501,112.95,9.502 ;$ $4.066,2.227,-5,9.282,-3,114.37,+11,9.281,-9 ; 4.146,2.085,9.092,115.97,9.088$; 4.232 , 1.942, 8.897, 117.83, 8,891; 4.327, 1.785,-4,8.688,-6, 120.12,+3,8.678,-6; $4.424,1.629,8.482,122.80,8.471 ; 4.528,1.473,8.269,125.89,8.258 ; 4.627,1.332,-4$, 8.073, - 11, 129.19, - 7, 8.058, -8; 4.734, 1.192, 7.866, 133.05, \%.848; 4.841, 1.066, 7.666, $137.15,7.645 ; 4.955,0.941,+3,7.456,-26,141.94,-5,7.433,-16 ; 5.038,0.858,7.304$, $145.59,7.285 ; 5.127,0.775,7.144,149.68,7.124 ; 5.205,0.707,+8,7.003,-35,153.30,+5$, $6.983,-26 ; 5.284,0.638,6.862,15 \% .20,6.846 ; 5.351,0.583,6.744,160.51,6.731 ; 5.419$, $0.529,+13,6.627,-35,163.94,-6,6.619,-37 ; 5.492,0.474,6.503,167.53,6.502 ; 5.544$, $0.434,6.420,170.26,6.418 ; 5.599,0.393,+9,6.327,-32,173.00,+1,6.330,-44 ; 5.652$, $0.353,6.242,175.82,6.249 ; 5.708,0.312,6.155,178.68,6.162 ; 5.800,0.245,6.016,183.49$, $6.025 ; 5.877,0.191,+3,5.905,-26,187.50,-19,5.916,-48 ; 5.959,0.138,5.791,191.65$, $5.802 ; 6.040,0.085,5.68,195.86,5.69 ; 6.103,0.045,5.60,199.13,5.61 ; 6.177,-0.001,+1$, $5.507,-20,203.18,-17,5.52_{3},-51 ; 6.258,-0.047,5.41,20 \% .35,5.43 ; 6.342,0.093$, $5.31,211.85, \quad 5.33 ; 6.435,-0.139,5.20,216.8_{2}, 5.22 ; 6.520,-0.179,+19,5.10_{0},-14$, $221.4_{9},-3,5.13_{2},-59 ; 6.613,-0.218,5.01,226.6,5.03 ; 6.698,-0.251,4.92,231.2,4.94$; $6.798,-0.283,4.81,236.4,4.84 ; 6.885,-0.309,4.72,241.4,4.75 ; 6.981,-0.335,4.63$, $246.6,4.65 ; 7.088,-0.361,252.6 ; 7.182,-0.381,257.9 ; 7.280,-0.400,263.1 ; 7.380$, $-0.419,268.7$;

$C_{\mathrm{A}}=1.91_{9} m M, E_{C_{\mathrm{M}}}=106.92 \mathrm{mV}: 2.944,3.517,107.66 ; 3.027,3.462,10 \% .69 ; 3.121$, $3.388,10 \% .76 ; 3.224,3.299,107.90 ; 3.327,3.207,-1,108.16,-1 ; 3.425,3.112,108.50$; $3.523,3.010,109.03 ; 3.619,2.899, \pm 0,109.69,+15 ; 3.713,2.784,110.59 ; 3.806,2.654$, $111.74 ; 3.901,2.512,+12,113.25,+10 ; 3.999,2.369,115.01 ; 4.042,2.307,115.86 ; 4.129$, $2.173,+9,117.91,+13 ; 4.216,2.044,120.16 ; 4.310,1.906,122.97 ; 4.416,1.763,+5$, $126.44,+11 ; 4.518,1.631,130.15 ; 4.643,1.490,134.85 ; 4.738,1.380,+8,139.19,+11 ;$ $4.849,1.271,144.19 ; 4.943,1.182,148.69 ; 5.046,1.094,+16,153.70,+4 ; 5.201,0.967$, $161.59 ; 5.264,0.919,164.69 ; 5.369,0.842,+21,170.04,-4 ; 5.458,0.774,174.65 ; 5.564$, $0.698,180.14 ; 5.656,0.632,+16,185.00,-26 ; 5.754,0.566,189.89 ; 5.844,0.500,194.82$; $5.942,0.434,+7,199.8_{5},-30 ; 6.044,0.369,205.1 ; 6.149,0.305,210.8 ; 6.248,0.249,+8$, $216.0_{5},-38 ; 6.355,0.195,221.9 ; 6.453,0.149,227.0 ; 6.538,0.113,+22,231.8_{5},-45$; $6.629,0.077,236.8 ; 6.733,0.041,242.3 ; 6.825,0.014,247.4 ; 6.921,-0.013,+49,252.5_{3},+7$; $7.026,-0.040,258.7 ; 7.122,-0.062,263.4 ; 7.229,-0.085,269.2 ; 7.726,-0.195,295.8$; $8.395,-0.400,326$;

Acta Chem. Scand. 19 (1965) No. 6 
$C_{\mathrm{A}}=3.044_{7} m, E_{C_{\mathrm{M}}}=107.03 \mathrm{mV}: 2.841,3.578,108.17 ; 2.917,3.528,108.19$; $2.995,3.474,108.23$; 3.107, 3.392, 108.35; 3.195, 3.324, 108.56; 3.280, 3.247, 108.89; $3.373,3.167, \pm 0,109.28,-16 ; 3.547,2.993,110.59 ; 3.630,2.901,111.56 ; 3.713,2.808,+1$, $112.71,+3 ; 3.790,2.713,114.06 ; 3.867,2.618,115.61 ; 3.965,2.492,+12,118.06,-9 ;$ $4.044,2.397,120.19 ; 4.133,2.287,123.04 ; 4.226,2.179,+7,126.27,-13 ; 4.310,2.086$, $129.45 ; 4.398,1.994,133.01 ; 4.488,1.903,-3,136.94,-9 ; 4.576,1.819,140.97,4.669$, $1.736,145.34 ; 4.767,1.653,-9,150.01,+5 ; 4.867,1.571,155.00 ; 4.968,1.490,160.16$; $5.062,1.418,-2,165.03,-2 ; 5.150,1.353,169.50 ; 5.256,1.274,175.05 ; 5.350,1.207,+4$, $179.87,-2 ; 5.443,1.140,184.61 ; 5.534,1.074,+3,189.40,-9 ; 5.631,1.004,194.59 ;$ $5.744,0.928,200.22 ; 5.848,0.854,205.92 ; 5.963,0.780,-12,211.90,-50 ; 6.032,0.738$, $215.45 ; 6.145,0.671,221.65 ; 6.286,0.599,-13,229.1,-60 ; 6.395,0.549$, 235.1 $; 6.506$, $0.503,241.1_{4} ; 6.616,0.463,+1,247.5,-101 ; 6.726,0.428,253.8 ; 6.829,0.399,260.0$; $6.944,0.369,266.8 ; 7.069,0.340,274.5 ; 7.177,0.315,285.7 ; 7.285,0.291,289 ; 7.686$, $0.185,316 ; 7.926,0.104,320$;

$$
C_{\mathrm{M}}=1.45_{5} m M
$$

$C_{\mathrm{A}}=1.01_{0} m M, \quad E_{C_{\mathrm{M}}}=101.52 \stackrel{m V}{m}: 3.082,3.380,102.06 ; 3.153,3.323,102.09$; $3.202,3.278,102.12 ; 3.286,3.197,102.21 ; 3.378,3.117,-3,102.35,-2 ; 3.458,3.010$, $102.49 ; 3.545,2.899,102.75 ; 3.631,2.777,103.06 ; 3.717,2.646, \pm 0,103.47,+9 ; 3.794$, $2.506,103.94 ; 3.872,2.363,104.48 ; 3.958,2.185,105.20 ; 4.056,1.985,+2,106.11,+6$; $4.150,1.782,107.10 ; 4.248,1.578,108.19 ; 4.346,1.373,109.35 ; 4.437,1.189,+8,110.48$, $-23 ; 4.532,1.005,111.67 ;(4.625,0.842,112.79 ; 4.719,0.690,113.89 ; 4.824,0.538,115.04$; $4.917,0.417,116.00 ; 4.978,0.336,116.67 ; 5.062,0.236,117.5 ; 5.166,0.116,118.4 ; 5.282$, $-0.004,119.4_{6} ; 5.390,-0.104,120.4 ; 5.499,-0.203,121.3_{5} ; 5.603,-0.293,122.3_{5} ; 5.717$, $-0.381,123.4 ; 5.811,-0.460,124.3 ; 5.906,-0.539,125.3 ; 6.018,-0.617,126 ;)$

$C_{\mathrm{A}}=1.45_{7} m M, E_{C_{\mathrm{M}}}=101.53 m V: 3.005,3.453,102.19 ; 3.079,3.392,12.202$, $102.21,12.203 ; 3.160,3.324,11.929,102.2 \%, 11.929 ; 3.246,3.246,+8,11.646,-11$, $102.37,-5,11.646,-11 ; 3.337,3.158,11.353,102.54,11.353 ; 3.430,3.058,11.061,102.78$, $11.063 ; 3.515,2.961,-7,10.803,-7,103.09,+6,10.804,-9 ; 3.584,2.866,10.599,103.44$, $10.601 ; 3.660,2.754,10.381,103.90,10.383 ; 3.745,2.626,-7,10.146,-1,104.50,+12$, $10.149,-8 ; 3.829,2.479,9.925,105.27,9.927 ; 3.913,2.330,9.713,106.16,9.716 ; 3.993$, $2.179,-6,9.519,+3,107.14,+11,9.524,-11 ; 4.078,2.012,9.323,108.34,9.326 ; 4.166$, $1.846,9.129,109.66,9.130 ; 4.251,1.680,-10,8.952,+12,111.13,+4,8.954,-5 ; 4.338$, $1.515,8.780,112.74,8.785 ; 4.430,1.350,8.609,114.49,8.609 ; 4.523,1.186,-12,8.446$, $+24,116.44,-16,8.444,+4 ; 4.621,1.024,8.285,118.62,8.280 ; 4.716,0.876,8.138$, $120.82,8.129 ; 4.807,0.742,-6,8.008,+35,122.94,-58,7.992,+21 ; 4.905,0.609,7.877$, $125.34,7.857 ; 4.996,0.490,7.76,127.69,7.73 ; 5.075,0.398,7.67,129.65$, \%.62; 5.190, $0.267,7.54,132.75,7.48 ; 5.293,0.163,7.43,135.50,7.35 ; 5.390,0.073,7.34,138.09$, $7.23 ; 5.489,-0.017,7.24,140.80,7.10 ; 5.591,-0.107,7.15,143.67,6.98 ;(5.686,-0.183$, $146.2_{0} ; 5.777,-0.259,148.8_{1} ; 5.882,-0.334,151.4_{6} ; 5.969,-0.397,153.6_{3} ; 6.057,-0.460$, $155.9 ; 6.147,-0.522,158.0 ; 6.244,-0.584,160.4 ; 6.352,-0.646,162.7 ; 6.447,-0.701$, $165.9 ; 6.557,-0.756,168.7 ; 6.668,-0.805,175_{.8} ; 6.883,-0.884,189.8 ; 7.105,-0.945$, $202 ; 7.257,-0.975,210$;)

$C_{\mathrm{A}}=1.90_{8} m M, E_{C_{\mathrm{M}}}=101.60 m V: 2.944,3.494,102.35 ; 3.010,3.450,102.34 ;$ $3.106,3.375,102.41 ; 3.208,3.287,+4,102.55,-7 ; 3.306,3.193,102.7 \% ; 3.403,3.094$, $103.10 ; 3.500,2.986,-8,103.51,+6 ; 3.590,2.869,104.10 ; 3.682,2.743,104.81 ; 3.771$, $2.608,-11,105.70,+11 ; 3.858,2.464,106.78 ; 3.948,2.309,108.10 ; 4.044,2.144,-13$, $109.69,+9 ; 4.136,1.979,111.44 ; 4.232,1.814,113.49 ; 4.329,1.651,-20,115.82,+7$; $4.434,1.484,118.58 ; 4.522,1.348,121.13 ; 4.606,1.229,-26,123.65,+6 ; 4.698,1.105$, $126.64 ; 4.797,0.983,130.1_{3} ; 4.898,0.866,-25,133.9_{5},+8 ; 4.997,0.760,137.9_{4} ; 5.095$, $0.665,142.1_{0} ; 5.189,0.579,-20,146.2_{0},+29 ; 5.289,0.494,150.8_{4} ; 5.390,0.414,155.5$; $5.480,0.344,159.9_{3} ; 5.573,0.275,-29,164.5_{4},+68 ; 5.666,0.206,169.2_{7} ; 5.759,0.137$, $174.3_{6} ; 5.862,0.068,179.3_{2} ; 5.958,0.005,-48,184.1_{7},+84 ; 6.054,-0.058,189.2 ; 6.157$, $-0.121,194.6 ; 6.247,-0.175,-48,199.5_{5},+65 ; 6.345,-0.228,204.7 ; 6.433,-0.272$, $209.4_{5} ; 6.531,-0.316,-35,214.7_{5},+73 ; 6.635,-0.360,220.6 ; 6.748,-0.399, \quad 226.5$; $6.843,-0.430,-10,232.0_{5},+60 ; 6.935,-0.456,238_{.0} ; 7.061,-0.486,244.5,7.189,-0.512$, $251 ; 7.331,-0.538,258 .{ }_{5} ; 7.726,-0.598,179 ; 8.099,-0.658,297$; 
$C_{\mathrm{A}}=3.05, m M, E_{C_{\mathrm{M}}}=101.57 m V: 2.828,3.580,102.60 ; 2.894,3.529,102.60$; $2.984,3.467,102.67 ; 3.084,3.391,102.80 ; 3.181,3.309,+9,103.01,-23 ; 3.279,3.220$, 103.34; 3.386, 3.116, 103.83; 3.482, 3.010, +1, 104.45, -5 ; 3.570, 2.904, 105.25; 3.664, $2.783,106.31 ; 3.759,2.652,+8,10 \% .69,+5 ; 3.850,2.520,109.32 ; 3.939,2.388,111.19$; $4.028,2.258,+15,113.35, \pm 0 ; 4.155,2.079,116.89 ; 4.243,1.961,119.68 ; 4.331,1.844,+9$, $122.80,-4 ; 4.427,1.730,126.43 ; 4.527,1.616,130.51 ; 4.636,1.504,-1,135.17,+5$; $4.750,1.394,140.45 ; 4.843,1.312,144.75 ; 4.936,1.231,+3,149.43, \pm 0 ; 5.035,1.151$, $154.35 ; 5.129,1.077,159.15 ; 5.226,1.004,+13,164.12,-6 ; 5.324,0.932,169.25 ; 5.417$, $0.865,174.01 ; 5.506,0.802,+11,178.60,-15 ; 5.601,0.734,183.55 ; 5.701,0.664,188.73$; $5.798,0.595,193.96 ; 5.898,0.527,-4,199.29,-59 ; 6.004,0.459,204.7 ; 6.104,0.397$, $210.1_{6} ; 6.204,0.340,-11,215.4_{1},-63 ; 6.299,0.289,220.66_{0} ; 6.406,0.238,226.2_{7} ; 6.514$, $0.191,+3,232.1_{5},-63 ; 6.632,0.146,238.8_{5} ; 6.742,0.109,244.9_{9} ; 6.833,0.082,+26$, 249.9 , $-79 ; 6.935,0.055,255.5 ; 7.043,0.028,261.7 ; 7.162,0.001,268.4 ; 7.307,-0.030$, $276.7 ; 7.689,-0.116,297$;

Nitric acid was prepared by diluting Merck's p.a. and was analysed by recrystallized potassium iodate.

Potassium hydroxide (EKA p.a.) was prepared according to Powell and Hiller ${ }^{40}$ and was stored under nitrogen. It was standardized with recrystallized potassium hydrogen phthalate. The KOH was also titrated with the nitric acid above with results that agreed within $0.2 \%$ of those obtained from the phthalate titrations.

Copper amalgam was prepared by electrolysis of a sulfuric acid copper(II) sulfate solution, using a mercury cathode and a platinum anode. The mercury (Grave) had been redistilled in vacuo. Copper(II) sulfate (Merck p.a.) was used without further purification. The amalgam was stored under dilute perchloric acid in an $\mathbf{N}_{\mathbf{2}}$ atmosphere. The copper(II) content of the amalgam was $3 \%$ (weight).

Triply distilled water, freshly prepared in a quartz apparatus, was used throughout.

\section{RESULTS AND CALCULATIONS}

The primary data, $\left(E_{m}, E_{g}, C_{\mathrm{H}}, C_{\mathrm{A}}, C_{\mathrm{M}}\right)$, in combination with $E_{g}^{\circ}, E c_{\mathrm{M}}$, and $E_{j}$ were used to calculate $\log h, \bar{q}, \eta$, from eqn. 1 and the relationships:

$$
\begin{aligned}
& \bar{q}=\left(C_{\mathrm{H}}-h\right) / C_{\mathrm{A}} \\
& \eta=E_{M} / 29.58
\end{aligned}
$$

Then the curves, $\bar{q}_{h}(\log h), \bar{q}(\log h)_{C_{\mathrm{A}}, C_{M}}$ and $\eta(\log h)_{C_{\mathrm{A}}, C_{\mathrm{M}}}$ were drawn.

Titrations were carried out for $C_{\mathrm{A}}$ values $0.7,1.0,1.5,2$, and $3 \mathrm{mM}$. For each $C_{\mathrm{A}}$, titrations were in general performed] with $C_{\mathrm{M}}$ equal to $0,0.4,0.7$, 1.0, and 1.5 mM. Every third point is given in the graphs of Figs. 1 and 2. The complete data are presented in Tables 1 and 2 . For the final refinement, using the least square program Letagrop, ${ }^{3} 187$ points were selected, which were as uniformly distributed as possible over the whole range (Table 2). The refinement of the $\mathrm{p} K$ involved 69 points from data $\bar{q}_{h}(\log h)_{C_{\mathrm{A}}}$. Titrations at high $C_{\mathrm{A}}$ were preferred because of the better precision obtained (Table 1).

The two sets of data were treated separately. By integration methods the free ligand concentration, $a$, and the contribution of the metal complexes, $C_{\mathrm{A} m}$, to the total ligand concentration, $C_{\mathrm{A}}$, were computed. The main species and preliminary equilibrium constants were then evaluated graphically. Finally, these constants were improved by Letagrop. ${ }^{3}$ 
Calculation of free ligand concentration, elimination of acid-base species

Equations for the calculation of $a$ and $C_{\mathrm{A} m}$ have been derived previously, ${ }^{29}$ using the approach indicated by Sillén. ${ }^{1}$ We may extend these equations by including in the "complexity sum", $S$, species with negative $q$ and $r \geqslant 0$. Expressions will also be derived to be used for the data $\eta(\log h)_{C_{\mathrm{A}}, C_{\mathrm{M}}}$. As a basis we will use the differential of the function, $S(m, h, a)$,

$$
\mathrm{d} S=\left(C_{\mathrm{A}}-a\right) \mathrm{d} \ln a+\left(C_{\mathrm{H}}-h\right) \mathrm{d} \ln h+\left(C_{\mathrm{M}}-m\right) \mathrm{d} \ln m
$$

transformed to

$$
\mathrm{d}\left(C_{\mathrm{A}} \ln a+C_{\mathrm{M}} \ln m-S-a-m\right)=\ln a \mathrm{~d} C_{\mathrm{A}}-\bar{q} C_{\mathrm{A}} \mathrm{d} \ln h+\ln m \mathrm{~d} C_{\mathrm{M}}
$$

1. Data $\bar{q}(\log h)_{C_{\mathrm{A}}}, C_{\mathrm{M}}$. By a further differentiation of eqn. 9 we may derive a relationship of mixed partial differentials from which we find by integration at constant $C_{\mathrm{A}}$ and $C_{\mathbf{M}}$

$$
\log a / C_{\mathbf{A}}=\log a_{0} / C_{\mathbf{A}}-\left[\int_{\log h_{0}}^{\log h}\left[\bar{q}+\left(\partial \bar{q} / \partial \ln C_{\mathbf{A}}\right)_{h, C_{\mathbf{M}}}\right] \mathrm{d} \log h\right]_{C_{\mathrm{A}}, C_{\mathbf{M}}}
$$

By definition

$$
\begin{aligned}
& C_{\mathrm{A}}=C_{\mathrm{A} h}+C_{\mathrm{A} m} \\
& C_{\mathrm{A} h}=a \sum_{q=0}^{4} \beta_{0 q 1} h^{q}=a \cdot f(h) \\
& \bar{q}_{h} C_{\mathrm{A} h}=a \sum_{q} \beta_{0 q 1} q h^{q}=\frac{a \cdot \mathrm{d} f(h)}{\mathrm{d} \ln h}
\end{aligned}
$$

Whence and

$$
\bar{q}_{h}=\mathrm{d} \ln f(h) / \mathrm{d} \ln h
$$$$
\mathrm{d} \log C_{\mathrm{A} h}=\mathrm{d} \log a+\mathrm{d} \log f(h)=\mathrm{d} \log a+\bar{q}_{h} \mathrm{~d} \log h
$$
$\log h$

$$
\log C_{\mathrm{A} h}=\log a / a_{0}+\int_{\log h_{0}}^{\log h} \bar{q}_{h} \mathrm{~d} \log h+\log C_{\mathrm{A} h_{0}}
$$

Inserting the expression for $\log a$ (eqn. 10) we get after adding $-\log C_{\mathrm{A}}$

$$
\log C_{\mathrm{A} h} / C_{\mathrm{A}}=\log C_{\mathrm{A} h_{0}} / C_{\mathrm{A}}+\left[\int_{\log h_{0}}^{\log h}\left[\bar{q}_{h}-\bar{q}-\left(\partial \bar{q} / \partial \ln C_{\mathrm{A}}\right)_{h}, C_{\mathrm{M}}\right] \mathrm{d} \log h\right]_{C_{\mathrm{A}}, C_{\mathrm{M}}}
$$

and finally $C_{\mathrm{A} m}$ by eqn. 11 .

The quantities $\log a / C_{\mathrm{A}}$ and $\log C_{\mathrm{A} h} / C_{\mathrm{A}}$ were solved graphically using eqns. 10 and 17. Through every set of experimental points, $\bar{q}(\log h)_{C_{\mathrm{A}}}, C_{\mathrm{M}}$, smooth curves were drawn. $C_{\mathrm{A}}$ was different for each curve, while $C_{\mathrm{M}}$ was the same (cf. Fig. 1). The curves were cut at intervals of $0.05 \log h$ units, starting with $-\log h=3.10$. For each $\log h, \bar{q}$ was read off and a set of curves $\bar{q}\left(\ln C_{\mathrm{A}}\right)_{h}, C_{\mathrm{M}}$ were drawn, from which the derivatives of eqns. 10 and 17 could be computed. The derivative was then plotted as a function of $\log h$ for each $C_{\mathrm{A}}$ so that its value could be obtained for each measured log $h$. Integration was done with intervals of $0.05 \log h$ units, using the trapezoid formula.

The integration constants, $\log a_{0} / C_{\mathrm{A}}$ and $\log C_{\mathrm{A} h_{v}} / C_{\mathrm{A}}$ (eqns. 10, 17), were calculated at $\log h=-3.10\left(\log h_{0}\right)$. In this point curves with the same $C_{\mathrm{M}}$ approximately coincide, and $\bar{q}$ differs only slightly from $\bar{q}_{h}$ (Fig. 1). This may indicate a low degree of complex 
formation involving mainly $\mathrm{MH}_{q} \mathrm{~A}$ species. Because of the acid dissociation constants obtained (Table 3), $\mathrm{MH}_{2} \mathrm{~A}$ was selected as the most likely complex in this $\mathrm{pH}$ range. The integration constants were calculated from the following equations

$$
\begin{aligned}
& a_{0} / C_{\mathrm{A}}=(\bar{q}-2) / \sum_{q=0}^{4}(q-2) \beta_{0 q 1} h^{q} \\
& C_{\mathrm{A} h_{0}} / C_{\mathrm{A}}=\left(a_{0} \sum_{q=0}^{4} \beta_{0 q 1} h^{q}\right) / C_{\mathrm{A}}
\end{aligned}
$$

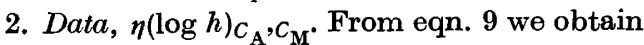

$$
\left(\frac{\partial \log a}{\partial C_{\mathrm{M}}}\right)_{h, C_{\mathrm{A}}}=\left(\frac{\partial \log m / C_{\mathrm{M}}}{\partial C_{\mathrm{A}}}\right)_{h, C_{\mathrm{M}}}
$$

Substituting $\log m / C_{\mathrm{M}}$ with $-E_{\mathrm{M}} / 29.58$ (eqn. 4), we find by integration at constant $h$ and $C_{\mathrm{A}}$

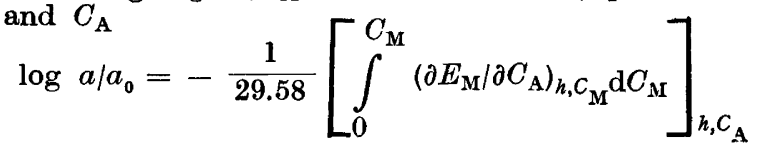

The integration constant, $\log a_{0}$, may be determined from a $\bar{q}_{h}(\log h)$ curve, using eqn. 16 rearranged to

$$
\log a_{0} / C_{\mathrm{A}}=\int_{\log h_{0}}^{\log h} \bar{q}_{h} \mathrm{~d} \log h+\log a_{00} / C_{\mathrm{A}}
$$

Since

$$
a_{00} / \mathrm{C}_{\mathrm{A}}=\left(\sum_{q=0}^{4} \beta_{0 q 1} h^{q}\right)^{-1}
$$

we may start the integration of eqn. 22 in any point of the log $h$ range. We preferred $\log h=-4.50$ since this point represents a high buffer capacity and is sufficiently removed from the first ionization of the phosphate (not considered in eqn. 23).

For the calculation of $\log a / a_{0}$ according to eqn. 21 experimental curves, $E_{\mathbf{M}}(\log h)_{C_{\mathrm{A}} C_{\mathrm{M}}}$, were cut at intervals of $0.10 \log h$ units in the range $\log h=-\mathbf{3 . 1 0}$ to -7.00 . Values of $E_{m}$ were read off and the derivative of eqn. 21 was determined from two neighboring intervals. In this calculation we considered some data at high $-\log h$, which were too uncertain to be used for the evaluation of equilibrium constants. These data are not shown in Fig. 2, but are given within parantheses in Table 2. The derivative of eqn. 21 was approximately constant at $\operatorname{low}-\log h$, while the quantity $C_{\mathrm{A}}\left(\Delta E_{\mathrm{M}} / \Delta \ln C_{\mathrm{A}}\right)_{h, C_{\mathrm{M}}}$ tended to be more constant in the higher range. The derivative was plotted against $C_{\mathrm{M}}$ for constant $h, C_{\mathrm{A}}$, and the curve obtained was extrapolated to $C_{\mathrm{M}}=0$. The area under the curve gave $\log a / a_{0}$. Families of curves $\log a / a_{0}(\log h)_{c_{\mathrm{A}}}, c_{\mathrm{M}}$ were then drawn so that $\log a / a_{0}$ could be obtained for each measured point.

The corresponding $\log a_{0}$ was determined by integration of the curve $\bar{q}_{h}(\log h)$ according to eqn. 22. The trapezoid formula was used with intervals of $0.05 \log h$ units.

The quantity, $C_{\mathrm{Am}}$, could easily be computed in this case using

and eqn. 11.

$$
C_{\mathrm{A} h} / C_{\mathrm{A}}=a / a_{0}
$$

Table 2 shows results obtained from every fourth titration at the computation of the free ligand concentration by eqns. 10 and 21 .

$$
\begin{aligned}
& \text { Prevailing values of } p, q, r \text { in the general formula } \\
& \mathbf{M}_{p} \mathbf{H}_{q} \mathbf{A} \text {, }
\end{aligned}
$$

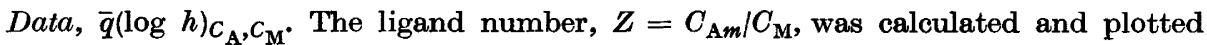
against $\log a$ for constant $h$. Up to $-\log h=4.90$ the curves were independent of $C_{\mathrm{M}}$ indicating that species $\mathrm{MH}_{q} \mathrm{~A}$, dominate. ${ }^{30}$ For higher $-\log h$ the curves fell within $Z=1.0$ to 1.2 and information about $p$ was instead obtained from $\bar{q}_{m}(\log h)$ curves.

Acța Chem. Scand. 19 (1965) No. 6 
These were independent of $C_{\mathrm{M}}$ in the whole log $h$ range, but depended slightly on $C_{\mathrm{A}}$ for $C_{\mathrm{A}}>1.5 \mathrm{mM}$. Apart from $\mathrm{MH}_{q} \mathrm{~A}$ there may thus be some $\mathrm{MH}_{q} \mathrm{~A}_{r}$ with $r \geqslant 2$.

To find $q$ and $r$ we considered the partial derivative

$(\partial \log a / \partial \log h)_{Z} \sim-q / r$

In the range $-\log h=3.1-4.9$ it varied from -2 to 0 . This agrees with the $\bar{q}_{m}(\log h)$ curves; $\bar{q}_{m}=\bar{q}_{0} / \bar{r}_{0}$ attained values between 2 to -1 in the $-\log h$ range 3.1 to 7 .

From mononuclearity follows that $Z=\bar{r}_{0}$, when $m<\left\langle C_{\mathrm{M}}\right.$. Since $Z \leqslant 1.2$ in the whole range, we assumed as a first approximation that $r_{\max }=2$. In the range $-\log h$ $<4.9$ this was checked by considering the plot $Z /(1-Z) a$ against $(2-Z) a /(1-Z)$ for constant $h$, using ${ }^{28}$

$$
\begin{aligned}
& Z /(1-Z) a=\sum_{q} \beta_{1 q_{1}} h^{q}+\sum_{q} \beta_{1 q_{2}} h^{q}(2-Z) a /(1-Z)=I+L(2-Z) a /(1-Z) \\
& \text { For }-\log h>4.9 \text { and } C_{\mathrm{A}}>1.5 \mathrm{mM},\left(m \ll C_{\mathrm{M}}\right), \text { we preferred } \\
& \left(C_{\mathrm{A} m}-C_{\mathrm{M}}\right) /\left(2 C_{\mathrm{M}}-C_{\mathrm{A} m}\right) a=\sum_{q} \beta_{1 q_{2}} h^{q} / \sum_{q} \beta_{1 q_{1}} h^{q}=f(h)
\end{aligned}
$$

and plotted the left hand side against $\log h$. The plots of eqn. 26 could be fitted to straight lines, while that of eqn. 27 was found to be independent of both $C_{\mathrm{A}}$ and $C_{\mathrm{M}}$. Thus, there is no reason to believe in species with $r>2$. The evidence for species $\mathrm{MH}_{q} \mathbf{A}_{2},(L \neq 0$ and $C_{\mathrm{A} m}-C_{\mathrm{M}}>0$ ), was found to be rather weak, specially at low $-\log h$.

In summary, the predominating species may conform to the following values of $p, q, r: p=1 ; r=1, q=2,1,0,-1 ; r=2, q=4,3,2,1,0,-1,-2$.

Data, $\eta(\log h)_{C_{\mathrm{A}}}, c_{\mathrm{M}}$. By considering curves $\eta_{0}(\log a)_{h}$ of the function

$$
\eta_{0}=\log \left(C_{\mathrm{M}}-m\right) / m=\log \sum_{p, q, r} p \beta_{p q r} m^{p-1} h^{q} a^{r}
$$

we reached the same main conclusion about prevailing $p, q, r$, as that arrived at for the $\operatorname{data}\left(\bar{q}, h, C_{\mathrm{A}}, C_{\mathrm{M}}\right)$.

Since species mononuclear in M predominate, ( $\eta$ was found to be a function of $h$ and $a$ only), we could use the partial derivatives

$$
\begin{aligned}
& (\partial \log a / \partial \log h)_{\eta_{0}} \sim-q / r \\
& \left(\partial \eta_{0} / \partial \log a\right)_{h} \sim{ }_{r}
\end{aligned}
$$

The former derivative was found to vary from -2 to 1 , while the latter remained approximately constant at unity. The main species may then be of type $\mathrm{MH}_{q} \mathrm{~A}$ with $q$ ranging from 2 to -1 .

When $C_{\mathrm{A}}>1.5 \mathrm{mM}$, however, there was evidence for some species $\mathrm{MH}_{q} \mathrm{~A}$, with $r>1$, since the quantity $C_{\mathrm{A} m} /\left(C_{\mathrm{M}}-m\right)=\bar{r}_{0}$ reached values up to 1.2 . To find $r_{\max }$ the equation

$$
\eta_{2}=\log \left(C_{\mathrm{A} m}-C_{\mathrm{M}}+m\right) / m a^{2}=\log \sum_{q, r}(r-1) \beta_{1 q_{2}} h^{q} a^{r-2}
$$

was used in the plot $\eta_{2}(\log h)$. Since $\eta_{2}$ was found to be a function of $h$ only, $r_{\max }=2$. The $q$ range for $r=2$, finally, was given by the derivative

$$
\mathrm{d} \eta_{2} / \mathrm{d} \log h \sim q
$$

It had the limiting values 4 and 0 . For these data we may thus exclude $q=-1,-2$, when $r=2$.

Table 3. Copper(II) complexes of O-phosphorylserylglutamic acid in $0.15 \mathrm{M} \mathrm{K}\left(\mathrm{NO}_{3}\right)$ medium at $25^{\circ} . \mathrm{p} K$ values of the ligand from graphical analysis and from Letagrop ${ }^{3}$ treatment.

\begin{tabular}{lllllr}
\hline Treatment & $\begin{array}{c}\mathrm{p} K\left(\mathrm{H}_{4} \mathrm{~A}\right) \\
\pm \mathbf{3} \sigma\end{array}$ & $\begin{array}{c}\mathrm{p} K\left(\mathrm{H}_{3} \mathrm{~A}\right) \\
\pm \mathbf{3} \sigma\end{array}$ & $\begin{array}{c}\mathrm{p} K\left(\mathrm{H}_{2} \mathrm{~A}\right) \\
\pm \mathbf{3 \sigma}\end{array}$ & $\begin{array}{c}\mathrm{p} K(\mathrm{HA}) \\
\pm \mathbf{3} \sigma\end{array}$ & $\begin{array}{r}1000 \\
\sigma\left(\bar{q}_{h}\right)\end{array}$ \\
\hline Letagrop & $\mathbf{3 . 0 5} \pm \mathbf{0 . 0 1}$ & $4.40 \pm 0.01$ & $\mathbf{5 . 6 9} \pm 0.01$ & $\mathbf{8 . 1 9} \pm 0.01$ & 5.7 \\
Graphical & $\mathbf{3 . 0 4}$ & $\mathbf{4 . 4 0}$ & $\mathbf{5 . 6 9}$ & $\mathbf{8 . 2 0}$ & \\
Graphical* & $\mathbf{3 . 0 2}$ & $\mathbf{4 . 3 9}$ & $\mathbf{5 . 6 9}$ & $\mathbf{8 . 2 5}$ & \\
\hline
\end{tabular}

* Ref. 39; $0.15 \mathrm{M}(\mathrm{K}) \mathrm{Cl}$ medium at $25^{\circ}$. 
Equilibrium constants

Acid dissociation constants, $K\left(\mathrm{H}_{q} \mathrm{~A}\right)$, were computed from the data, $\left(\bar{q}_{h}\right.$, $\log h)_{c_{\mathrm{A}}}$, as described previously. ${ }^{39}$ The result is shown by Table 3 . The $\bar{q}_{h}(\log \mathrm{h})$ curve for $C_{\mathrm{A}}=0.7 \mathrm{mM}$ deviated significantly for $-\log h>5$, being maximally $0.02 \bar{q}_{h}$ units at $\mathrm{pH}$ 7. The deviation observed may be explained by the presence of an impurity, e.g. carbonate. We tried to correct for this by making calculations for this $\bar{q}_{h}(\log h)$ curve separately.

Data, $\bar{q}(\log h)_{C_{\mathrm{A}}, C_{M}} ;$ species $\mathrm{MH}_{q} \mathrm{~A}$. In the $-\log h$ range 3.1 to 4.9 we used the ordinate intercepts, $I$, of eqn. 26 to form the function ${ }^{28}$

$$
I=\sum_{q} \beta_{1 q 1} h^{q}
$$

Species $\mathrm{MH}_{-1} \mathrm{~A}$ was neglected in this range, and we summed for $q=2,1,0$; $\left(\bar{q}_{m}>0.4\right.$ for $\left.-\log h<4.9\right)$.

To find the $\beta_{1 q 1}$ the $\log I(\log h)$ curve was compared to a family of normalized curves $\mathbf{Y}(\log v)_{p}$ of the function ${ }^{41}$ (cf. the monograph ${ }^{42}$ )

$$
\mathbf{Y}=\log \left(v^{2}+v p+1\right)
$$

where

$$
\begin{array}{ll}
\log I=\log \beta_{101} & \text { for } \mathbf{Y}=0 \\
-\log h=1 / 2 \log \beta_{121} / \beta_{101} & \text { for } \log v=0
\end{array}
$$

$\log p=\log \beta_{111}-1 / 2 \log \beta_{121} \beta_{101}$

From the best fit we obtained the preliminary constants: $\log \beta_{121}=16.63$, $\log \beta_{111}=13.03, \log \beta_{101}=8.37$.

Since the plot $\vec{q}_{m}(\log h)$ was found to be independent of $C_{\mathrm{A}}$ for $C_{\mathrm{A}} \leqslant 1.5 \mathrm{mM}$, we may assume that the system is monoligandic in this range. For $-\log h>4.8$ we also neglected species with $\bar{q} \geqslant 2,\left(\bar{q}_{m}<0.5\right)$, and considered the limiting function

$$
\bar{q}_{m_{1}}=\frac{\beta_{111} h-\beta_{1-11} h^{-1}}{\beta_{111} h+\beta_{101}+\beta_{1-11} h^{-1}}
$$

The curve $q_{m_{1}}(\log h)$ was compared to normalized graphs $y(\log v)_{p}$, where

$$
\begin{array}{ll}
y=\frac{v-v^{-1}}{v+p+v^{-1}} & \\
-\log h=1 / 2 \log \beta_{111} / \beta_{1-11} & \text { for } \log v=0 \\
\log p=\log \beta_{101}-1 / 2 \log \beta_{111} \beta_{1-11} &
\end{array}
$$

This gave

$$
\log \beta_{101}-\log \beta_{1-11}=\mathrm{p} K(\mathrm{MA})=5.97 ; \log \beta_{111}-\log \beta_{101}=4.64 .
$$

The latter difference agrees with that $(=4.66)$ of the same constants evaluated from the $\log I(\log h)$ graph.

Species $\mathrm{MH}_{q} \mathrm{~A}_{2}$. The following function was formed

$$
Y_{2}=\sum_{q} \beta_{1 q 2} h^{q_{-2}}
$$

Acta Chem. Scand. 19 (1965) No. 6 
When $-\log h<4.9$, values of $Y_{2}$ was computed from ${ }^{28}$

$$
h^{-2} L=\sum_{192} \beta_{1} h^{q-2}
$$

Values on $L$ are obtained from the slopes of the eqn. 26 lines. In the range $-\log h>5.0$ we computed $Y_{2}$ by eqn. 27 rearranged to

$$
\left(\sum_{q=-1}^{2} \beta_{1 q 1} h^{q-2}\right)\left(C_{\mathrm{A} m}-C_{\mathrm{M}}\right) /\left(2 C_{\mathrm{M}}-C_{\mathrm{A} m}\right) a=\sum_{q} \beta_{1 q 2} h^{q-2}
$$

using the $\beta_{1 q 1}$ previously obtained (see above).

By comparing the curve $\log Y_{2}(2 \log h)$ with normalized graphs it was found that we had to consider at least three species $\mathrm{MH}_{q} \mathrm{~A}_{2}$ with $q$ equal to $4,2,0$ or $4,1,0$. The first combination gave the best fit. Using a family of curves $\mathbf{Y}(\log v)_{p}$ of the function ${ }^{41}$

$$
\mathbf{Y}=\log \left(v+p+v^{-1}\right)
$$

where

$\log Y_{2}=1 / 2 \log \beta_{142} \beta_{102}$

$-2 \log h=1 / 2 \log \beta_{142} / \beta_{102}$

$\log p=\log \beta_{122}-1 / 2 \log \beta_{142} \beta_{102}$ for $\mathbf{Y}=0$

for $\log v=0$

we obtained

$$
\log \beta_{142}=33.3 ; \log \beta_{122}=24.4 ; \log \beta_{102}=12.6 \text {. }
$$

A set of four species, $(1,4,2),(1,2,2),(1,1,2),(1,0,2)$, also gave a satisfactory fit with normalized curves, while a combination with $\mathrm{MH}_{3} \mathrm{~A}_{2}$ could be rejected.

The data represented as a $\log Y_{2}(2 \log h)$ curve showed a spread of \pm 0.3 units on the ordinate. These data together with those of eqns. 26 and 27 (cf. p. 1460) thus jindicate that the species $\mathrm{MH}_{q} \mathrm{~A}_{2}$ are not very important in the present $C_{\mathrm{A}}$ range. Valid conclusions about such species may require measurements at higher $C_{\mathrm{A}} \cdot *$

Data $\eta(\log h)_{C_{\mathrm{A}}, C_{\mathrm{M}}} ;$ species $\mathrm{MH}_{q} \mathrm{~A}$. In the range $-\log h<4.7$, we neglected $\mathrm{MH}_{-1} \mathrm{~A}$ and compared the curve $\eta_{1}(\log h)$ of the function (cf. eqn. 28)

$$
\eta_{1}=\log \left(2 \times 1 \eta^{\eta_{\bullet}}-m^{-1} C_{\mathrm{Am}}\right) a^{-1}=\log \left(\sum_{q=0}^{2} \beta_{1 q 1} h^{q} \mathrm{II}\right)
$$

with normalized graphs $y(\log v)_{p}$ of eqn. 34. This gave $\log \beta_{121}=16.57$, $\log \beta_{111}=13.08, \log \beta_{101}=8.35$.

Then $* \eta_{1}(\log h)$ data computed from

$$
* \eta_{1}=\log \left(10^{\eta_{1}}-\beta_{121} h^{2}\right)=\log \left(\beta_{111} h+\beta_{101}+\beta_{1-11} h^{-1}\right)
$$

were compared to normalized curves $Y(\log v)$ of eqn. 40 (Fig. 3), using the preliminary $\beta_{121}$. Since

$$
\begin{array}{ll}
* \eta_{1}=1 / 2 \log \beta_{111} \beta_{1-11} & \text { for } \mathbf{Y}=0 \\
-\log h=1 / 2 \log \beta_{111} / \beta_{1-11} & \text { for } \log v=0 \\
\log p=\log \beta_{101}-1 / 2 \log \beta_{111} \beta_{1-11} &
\end{array}
$$

* Such measurements could not be carried out because of the limited amount of peptide available (about $500 \mathrm{mg}$ ). 


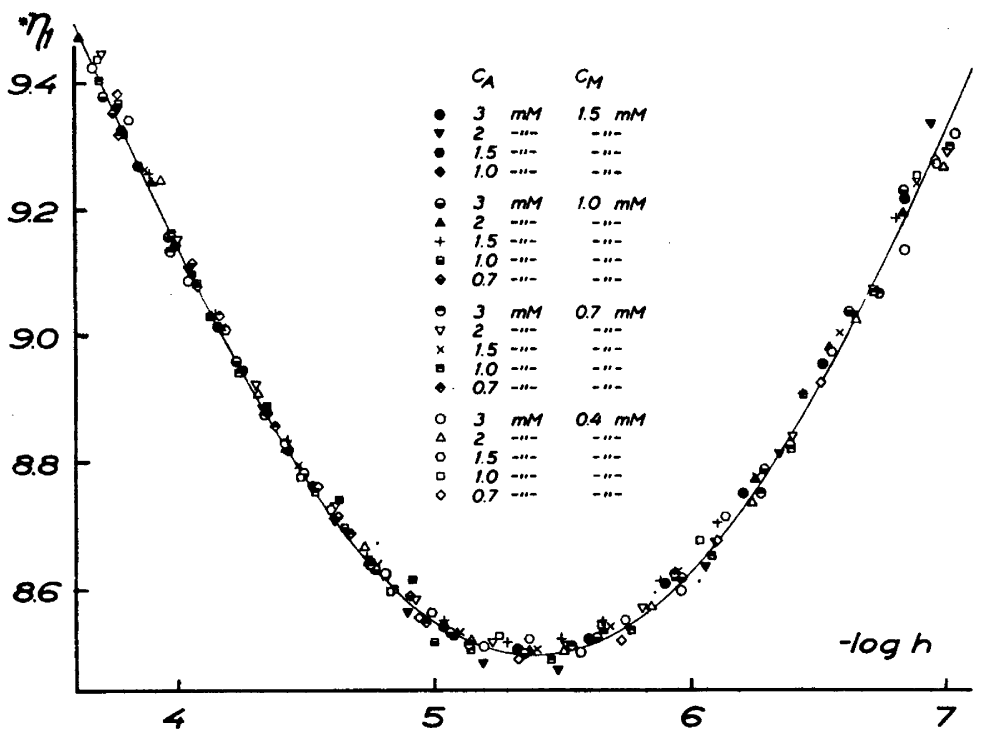

Fig. 3. Copper(II) complexes of phosphorylserylglutamic acid. ${ }^{*} \eta_{1}$ as a function of $-\log h$ according to eqn. 42. Drawn curve represents normalized curve, calculated from eqn. 40 , in position of the best fit and giving $\log \beta_{111}=13.07, \log \beta_{101}=8.34, \log \beta_{1-11}=2.29$.

we obtained: $\log \beta_{111}=13.07, \log \beta_{101}=8.34$,

$\log \beta_{1-11}=2.29,\left(\log \beta_{101} / \beta_{1-11}=\mathrm{p} K(\mathrm{MA})=6.05\right)$.

Species $\mathrm{MH}_{q} \mathrm{~A}_{2}$. Experimental data, $\log \eta_{2}-2 \log h$ against $2 \log h$ according to eqn. 31, were compared to a family normalized graphs $\mathbf{Y}(\log v)_{p}$ of eqn. 40. In the position of the best fit the result was: $\log \beta_{142}=33.2, \log \beta_{122}=$ $24.3, \log \beta_{102}=12.5$.

Other sets of complexes were also tried. The set $(1,4,2),(1,2,2),(1,1,2)$, $(1,0,2)$ gave a reasonable fit, while $(1,4,2),(1,2,2),(1,1,2)$ agreed up to $-\log h=6$. Combinations with $(1,3,2)$ could be rejected.

\section{Refinement of equilibrium constants}

The refinement was performed by the generalized least square method described by Sillén, ${ }^{2}$ using the program Letagrop. ${ }^{3}$ The high speed computer, SAAB D21, was used and Letagrop was rewritten in the D21 autocode (DAC). Since the main species apparently conform to the formula $\mathrm{MH}_{q} \mathrm{~A}_{r}, m$ could be eliminated and only the free ligand concentration, $a$, had to be computed. For the matrix inversion there was no standard program available in DAC. Therefore, we preferred to keep the symmetrical matrix (cf. 12/I in Table 5 of Ref. 3) triangular and carry out the inversion according to Cholesky-Rubin. 
The computer refines a guessed value of $a$ by the equation

$$
\begin{aligned}
& C_{\mathrm{A}(\text { calc })}=C_{\mathrm{A} h}+C_{\mathrm{A} m}=a \sum_{q=0}^{4} \beta_{0 q 1} h^{q}+C_{\mathrm{M}} Z_{\mathrm{calc}}= \\
& =a \sum_{q=0}^{4} \beta_{0 q 1} h^{q}+\frac{C_{\mathrm{M}} \sum_{q, r} \beta_{1 q_{r} r} r h^{q} a^{r}}{1+\sum_{q, r} \beta_{1 q r} h^{q} a^{r}}
\end{aligned}
$$

until $C_{\mathrm{A}(\text { calc })}$ fulfills the condition

$$
\left|C_{\mathrm{A}(\exp )}-C_{\mathrm{A}(\text { calc })}\right| \leqslant 0.0001 C_{\mathrm{A}(\exp )}
$$

From the amalgam data ${ }^{43}$ the machine determines $E_{m \text { (calc) }}$ by the equation

$$
E_{m(\text { calc })}=E_{C M}+29.577 \log C_{M} / m=E_{C M}+29.577 \log \left(1+\sum_{q, r} \beta_{1 q r}{ }^{q} h a^{r}\right)
$$

and the error square sum, $U_{m}$, from

$$
U_{m}=\sum\left(E_{m(\text { calc })}-E_{m}-E_{j}\right)^{2}
$$

From data, $\left(q, \log h, C_{\mathbf{A}}, C_{\mathbf{M}}\right)$, the error square sum, $U_{g}$, is computed by

$$
U_{\mathrm{g}}=\sum\left(\bar{q}_{\text {(calc) }}-\bar{q}\right)^{2}
$$

where

$$
\bar{q}_{\mathrm{calc}}=\frac{a \sum_{q=1}^{4} \beta_{0 q 1} q h^{q}+C_{\mathrm{M}} Z_{\text {calc }} \bar{q}_{m \mathrm{calc}}}{a \sum_{q=0}^{4} \beta_{0 q 1} h^{q}+C_{\mathrm{M}} Z_{\mathrm{calc}}}
$$

and

$$
\bar{q}_{m \text { (calc) }}=\frac{\sum_{q, r} \beta_{1 q,} q h^{q} a^{r}}{\sum_{q, r} \beta_{1 q_{r}} r h^{q} a^{r}}
$$

In the refinement of $\mathrm{p} K\left(\mathrm{H}_{q} \mathrm{~A}\right)$ eqns. 47 and 48 were used. The quantity $\bar{q}_{\text {(calc) }}$ (properly $\bar{q}_{h(\text { calc) }}$ ) was obtained directly from values on $h$ and $\beta_{0 q 1}$ since it is independent of $a$. The final results are shown by Table 3. The deviation $10^{3}\left(\bar{q}_{h(\text { calc })}-\bar{q}\right)$ of the 69 points selected is indicated in Table 1.

The Letagrop results on the metal complex equilibria are shown in Table 4. The values for $10^{3}\left(\bar{q}_{(\text {calc })}-\bar{q}\right)$ and $10^{2}\left(E_{m(\text { calc })}-E_{m}-E_{j}\right)$ of the 187 points selected are compiled in Table 2 . The computer varied the quotients indicated in Table 4 . The starting values were calculated using the $\beta_{1 q_{r}}$ obtained graphically. The $\beta_{0 q 1}$ were obtained from the refined $\mathrm{p} K$, which were kept constant in all runs. The variation of the constants were successively decreased. and repeated until constancy.

As can be seen from Table 4 species $\mathrm{MA}$ and $\mathrm{MH}_{-1} \mathrm{~A}$ come out with welldefined constants. The errors in $\beta_{111} / \beta_{011}$ and $\beta_{121} / \beta_{021}$ indicate that it is certainly necessary to assume $\mathrm{MHA}$ and $\mathrm{MH}_{2} \mathrm{~A}$, too. The introduction of two $\mathrm{MH}_{q} \mathrm{~A}_{2}$ species, $(1,2,2)$ and $(1,0,2)$, improved the agreement with experimental data; $c f . \sigma\left(E_{m}\right)$ and $1000 \sigma(\bar{q})$. The calculations involved alternative 


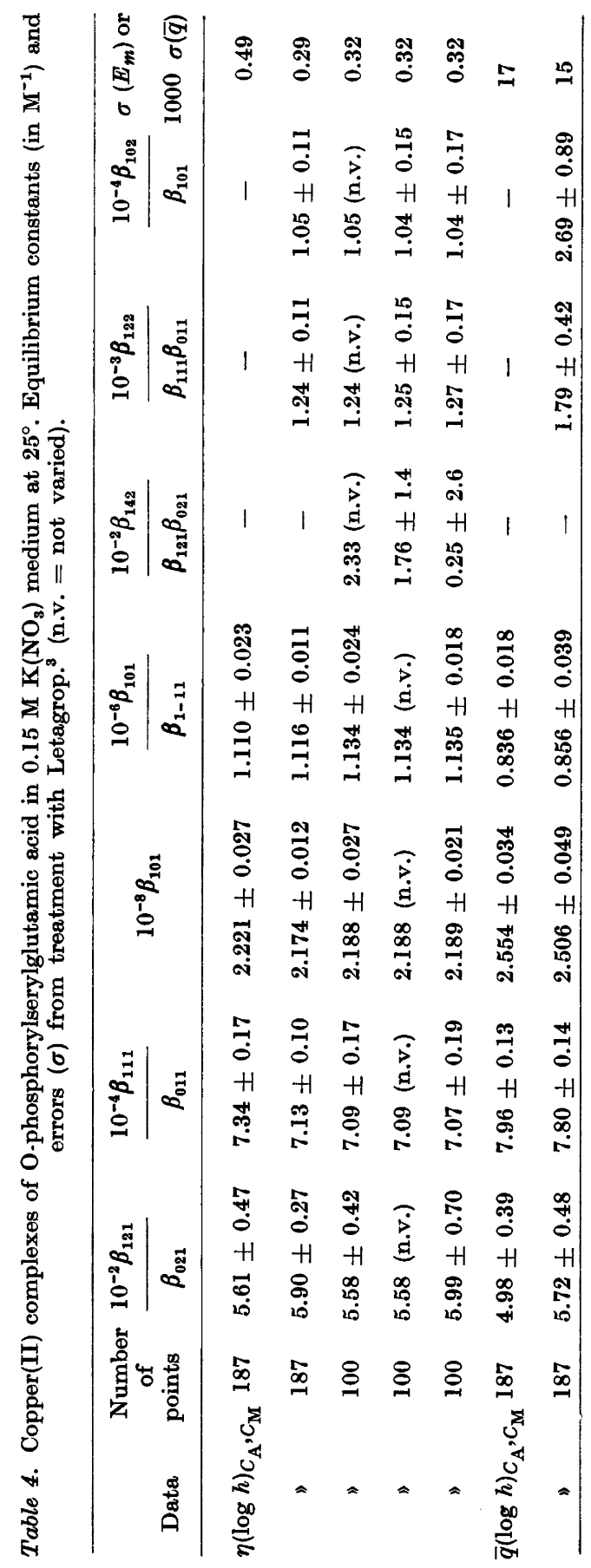

Acta Chem. Scand. 19 (1965) No. 6 
variations of the constants related to $r=1$ and $r=2$ followed by variations of all the six constants. The last sets for the amalgam data include $(1,4,2)$. The error in $\beta_{142} / \beta_{121} \beta_{021}$, obtained for constant or varying $\beta_{1 q 1}$, gives, however, no support for this species. A combination with four $\mathrm{M}_{q} \mathrm{HA}_{2},(1,4,2),(1,2,2)$, $(1,1,2),(1,0,2)$, was tried with the same data, but there was no improved agreement.

Consequently we selected sets (2) and (7) as the "best" sets. After conversion to $\log \beta_{p q_{r}}$ and $\mathrm{p} K(\mathrm{MA})$ and giving $3 \sigma\left(\log \beta_{p q_{r}}\right)$ and $3 \sigma(\mathrm{p} K(\mathrm{MA}))$ set (2) becomes

$\log \beta_{121}=16.65 \pm 0.12, \log \beta_{111}=13.04 \pm 0.05, \log \beta_{101}=8.34 \pm 0.01$, $\mathrm{p} K(\mathrm{MA})=6.05 \pm 0.02, \log \beta_{122}=24.3 \pm 0.2, \log \beta_{102}=12.36 \pm 0.14$.

From sets (2) and (7) the computer calculated the curves $\eta(\log h)_{c_{\mathrm{A}}, c_{\mathrm{M}}}$ and $\bar{q}(\log h)_{c_{\mathrm{A}}, c_{\mathrm{M}}}$, respectively, using the program Kuska. ${ }^{3}$ For comparison $\bar{q}(\log h)$ curves were also calculated by set $(2)$.

The original version of Kuska was rewritten for species $\mathrm{MH}_{q} \mathrm{~A}_{\text {, into the }}$ D21 autocode. An XY-writer was adapted to the computer so that curves could be obtained at the same time as data were punched out. (The program was worked out in collaboration with $\mathrm{S}$. Gobom). The results are shown by Figs. 1 and 2.

\section{Systematic errors}

It follows from sets (2) and (7) of Table 4 that we cannot exclude systematic errors in the present data. The refined $\beta_{101}$ and $\beta_{101} / \beta_{1-11}$ are slightly outside the $3 \sigma$ range. Apart from errors in $C_{\mathrm{A}}, C_{\mathrm{M}}$ and total [OH], uncertainties in $E^{\circ}$ and $\mathrm{p} K$ values may contribute to this result. In addition, a certain amount of $\mathrm{Cu}^{+}$formed in contact with the amalgam may give a considerable error, which might be serious at low $C_{\mathrm{M}}$ (Ref. 44). This process might, however, be included in the random errors of each titration, since the curves of Fig. 1 could be reproduced satisfactorily in the absence of amalgam.

The constants obtained from the amalgam data have lower standard deviations and give a better agreement with the experiments. One reason for this may be that analytical errors and minor impurities contribute more to $\vec{q}$ than to $\eta$. Assuming $0.5 \%$ error in $C_{\mathrm{A}}$ and total [OH], (analytical error $\pm 0.2 \%$ ) the deviation in $\bar{q}$ will be 0.03 to 0.06 units in the $\bar{q}$ range 2 to -1 . Fig. 1 shows that the amalgam set gives $\bar{q}(\log h)_{c_{\mathrm{A}}, c_{\mathrm{M}}}$ curves, which are within this deviation.

\section{DISCUSSION}

The two types of measurements carried out on the present system show independently that the predominating species in the concentration ranges investigated are of type $\mathrm{MH}_{q} \mathrm{~A}$. In the high log $h$ range, true conclusion about any particular species could only be reached from measurements made by the amalgam half-cell. The data, $\bar{q}(\log h)_{C_{\mathrm{A}}, C_{\mathrm{M}}}$, cannot be used to detect complexes that involve the complete protonized form of the ligand, $\left(\mathrm{H}_{5} \mathrm{~A}\right)$. The accuracy in $\bar{q}$ will also decrease for increasing $h$, until $\bar{q}$ becomes a small difference between two big quantities (cf. Ref. ${ }^{45}$ ). It was found, however, that measur- 
able complex formation started with species $\mathrm{H}_{2} \mathrm{~A}$, i.e. far from the $\mathrm{H}_{5} \mathrm{~A}$ species. These results agree with those obtained on the corresponding $\mathrm{Ca}, \mathrm{Mg}$ and $\mathrm{Mn}$ (II) systems. $^{29}$

The existence of the complex $\mathrm{MH}_{-1} \mathrm{~A}$ is uniquely demonstrated by the amalgam measurements. Products of copper(II) hydrolysis, ${ }^{36,37} \mathrm{MOH}$ and $\mathrm{M}_{2}(\mathrm{OH})_{2}$, may have consumed additional amounts of potassium hydroxide, but such species should have given a trend with $C_{\mathrm{A}}$ and $C_{\mathrm{M}}$ in the Fig. 3 plot. The $\mathrm{p} K(\mathrm{M} A \mathrm{~A})$ value of 6.05 indicates that the proton liberated comes from the peptide bond rather than from a coordinated water molecule. With this ligand the reaction occurs, however, at a more alkaline $\mathrm{pH}$ than with glycylglycine $(\mathrm{p} K(\mathrm{MA}) \sim 4)$. This may by caused by the stable MA complex obtained by phosphorylation ( $\log \beta_{1}=8.34 ; c f$. Refs. $7,10,15,16,23, \log \beta_{1}$ (glycylglycine) $\sim$ 5.5). A probable structure of this complex may be represented by<smiles>O=C1CCC(C(=O)[O-])NC(=O)C2COP3(=O)NC(O1)(O2)O3</smiles>

which is sterically possible according to a molecular model. The filling of the coordination positions of copper(II) may weaken its attack on the peptide bond and result in the higher $\mathrm{p} K(\mathrm{MA})$ value.

Chelate formation may be involved also in the other $\mathrm{MH}_{q} \mathrm{~A}$ species. The two carboxyl groups may complex in species $\mathrm{MH}_{2} \mathrm{~A}$, the phosphate and $\alpha$-carboxyl groups in MHA (cf. formula I, Fig. 6 in Ref. 29), and amino, amide, phosphate or/and carboxyl groups in $\mathrm{MH}_{-1} \mathrm{~A}$. Such multidentate complex formation may be the reason for the weak tendency of the peptide to form diligandic species. In this respect the phosphoryl group is surpassed by the imidazole group, since only monoligandic copper(II) species have been detected in solutions of histidyl peptides. ${ }^{7,17,22}$

The $\mathrm{p} K$ for the proton equilibria of the complexes, $\mathrm{p} K\left(\mathrm{MH}_{2} \mathrm{~A}\right)=2.3$, $\mathrm{p} K(\mathrm{MHA})=5.0, \mathrm{p} K(\mathrm{MA})=6.0$, indicate that species $\mathrm{MH}_{-1} \mathrm{~A}$ dominate in the neutral $\mathrm{pH}$ range. This is also valid for peptides like glycylglycine. ${ }^{23}$ The main binding groups should thus be the same (amino and amide groups) at $\mathrm{pH}$ of biochemical interest. However, the phosphorylpeptide differs from other peptides in that it can occupy some more coordination positions on the metal ion. This is valid not only for the copper(II) species $\mathrm{MA}$ and $\mathrm{MH}_{-1} \mathrm{~A}$ but also for the MA species formed by $\mathrm{Ca}^{2+}, \mathrm{Mg}^{2+}$, and $\mathrm{Mn}^{2+}$ ions. ${ }^{29}$ From a catalytic point of view the result may be that the metal ions become inactive. ${ }^{46}$ It has also been observed that phosphorylation decreases the susceptibility of a peptide to leucine aminopeptidase, which is activated by $\mathrm{Mg}^{2+}$ and $\mathrm{Mn}^{2+}$ ions ${ }^{47-49}$ (cf. the review ${ }^{50}$ ). Consequently, an unfavourable metal ion-substrate complex may be one reason for the decreased susceptibility. Moreover, the greater metal ion binding capacity of the substrate obtained by phosphorylation indicates that the optimum of $\mathrm{pM}$ should be reconsidered. 
Acknowledgements. I wish to thank Professor Ido Leden and Fil.lic. Sylvia Gobom for valuable discussions. My thanks are also due to Dr. G. Fölsch for the gift of $\mathrm{O}$ phosphorylserylglutamic acid and to Mr. Bo Sjöberg for skilful technical assistance.

This work has been financially supported by the Scientific Council of the Swedish Dairies Association and by the Swedish Medical Research Council.

\section{REFERENCES}

1. Sillén, L. G. Acta Chem. Scand. 15 (1961) 1981.

2. Sillén, L. G. Acta Chem. Scand. 16 (1962) 159.

3. Ingri, N. and Sillén, L. G. Acta Chem. Scand. 16 (1962) 173.

4. Monk, C. B. Trans. Faraday Soc. 47 (1951) 285.

5. Evans, P. W. and Monk, C. B. Trans. Faraday Soc. 51 (1955) 1244.

6. Li, N. C. and Doody, E. J. Am. Chem. Soc. 76 (1954) 221.

7. Dobbie, H. and Kermack, W. O. Biochem. J. 59 (1955) 246.

8. Dobbie, H. and Kermack, W. O. Biochem. J. 59 (1955) 257.

9. Manyak, A.R., Murphy, C.B. and Martell, A.E. Arch. Biochem. Biophys. 59 (1955) 373.

10. Datta, S. P. and Rabin, B. R. Trans. Faraday Soc. 52 (1956) 1123.

11. Rabin, B. R. Trans. Faraday Soc. 52 (1956) 1130.

12. Murphy, C. B. and Martell, A. E. J. Biol. Chem. 226 (1957) 37.

13. Rabin, B. R. Biochem. Soc. Symp. No. 15 (1958) 21.

14. Datta, S. P., Leberman, R. and Rabin, B. R. Trans. Faraday Soc. 55 (1959) 2141.

15. Koltun, W. L. and Gurd, F. R. N. J. Am. Chem. Soc. 81 (1959) 301.

16. Biester, J. L. and Ruoff, P. M. J. Am. Chem. Soc. 81 (1959) 6517.

17. Martin, R. B. and Edsall, J. T. J. Am. Chem. Soc. 82 (1960) 1107.

18. Koltun, W. L., Fried, M. and Gurd, F. R. N. J. Am. Chem. Soc. 82 (1960) 233.

19. Strandberg, B., Lindqvist, I. and Rosenstein, R. Z. Krist. 116 (1961) 266.

20. Cooper, T., Freeman, H. C., Robinsson, G. and Schoone, J.'C. Nature 194 (1962) 1237.

21. Koltun, W. L., Roth, R. H. and Gurd, F. R. N. J. Biol. Chem. 238 (1963) 124.

22. Doran, M. A., Chaberek, S. and Martell, A. E. J. Am. Chem. Soc. 86 (1964) 2129.

23. Kim, M. K. and Martell, A: E. Biochemistry 3 (1964) 1169.

24. Tschugaeff, L. Ber. 40 (1907) 1973.

25. Greenwald, I. J. Phys. Chem. 47 (1943) 607.

26. Rosenberg, A. Acta Chem. Scand. 11 (1957) 1390.

27. Österberg, R. Arkiv Kemi 13 (1959) 393.

28. Österberg, R. Acta Chem. Scand. 14 (1960) 471.

29. Österberg, R. Acta Chem. Scand. 16 (1962) 2434.

30. Leden, I. Potentiometrisk undersökning av några kadmiumsalters komplexitet, Diss., Lund 1943.

31. Biedermann, G. and Sillén, L. G. Arkiv Kemi 5 (1953) 425.

32. Meyer, F. R. and Ronge, G. Z. angew. Chem. 52 (1939) 637.

33. Forsling, W., Hietanen, S. and Sillén, L. G. Acta Chem. Scand. 6 (1952) 901.

34. Brown, A. S. J. Am. Chem. Soc. 56 (1934) 646.

35. Gran, G. Analyst 77 (1952) 661.

36. Berecki-Biedermann, C. Arkiv Kemi 9 (1955) 175.

37. Pedersen, K. J. Kgl. Danske Videnskab. Selskab, Mat.-Fys. Medd. 20 (1943) No. 7.

38. Fölsch, G. Acta Chem. Scand. 13 (1959) 1407.

39. Fölsch, G. and Österberg, R. Acta Chem. Scand. 15 (1961) 1963.

40. Powell, J. E. and Hiller, M. A. J. Chem. Educ. 34 (1957) 330.

41. Sillén, L. G. Acta Chem. Scand. 10 (1956) 186.

42. Rossotti, F. J. C. and Rossotti, H. The determination of stability constants, London 1961 .

43. Gobom, S. Acta Chem. Scand. 17 (1963) 2181.

44. Fronæus, S. Komplexsystem hos koppar, Diss., Lund 1948.

45. Biedermann, G. Svensk Kem. Tidskr. 76 (1964) 362.

46. Langenbeck, W., Mix, H. and Tittelbach-Helmrich, W. Chem. Ber. 90 (1957) 2699.

47. Fischer, E. H., Graves, D. J., Crittenden, E.R.S. and Krebs, E. G. J. Biol. Chem. 234 (1959) 1698.

48. Österberg, R. Acta Chem. Scand. 18 (1964) 795.

49. Fölsch, G., Strid, L. and Mellander, O. Acta Chem. Scand. 19 (1965) 1566.

50. Perlmann, G. E. Advan. Protein Chem. 10 (1955) 1. 